\title{
Symmetry, Thermodynamics, and Topology in Active Matter
}

\author{
Mark J. Bowick $\odot,{ }^{1}$ Nikta Fakhri $\odot,{ }^{2}$ M. Cristina Marchetti $\odot,{ }^{3}$ and Sriram Ramaswamy ${ }^{4}$ \\ ${ }^{1}$ Kavli Institute for Theoretical Physics, University of California Santa Barbara, \\ Santa Barbara, California 93106, USA \\ ${ }^{2}$ Department of Physics, Massachusetts Institute of Technology, Cambridge, Massachusetts 02139, USA \\ ${ }^{3}$ Department of Physics, University of California Santa Barbara, Santa Barbara, California 93106, USA \\ ${ }^{4}$ Centre for Condensed Matter Theory, Department of Physics, \\ Indian Institute of Science, Bangalore 560 012, India
}

(Received 30 June 2021; published 11 February 2022)

\begin{abstract}
The name active matter refers to any collection of entities that individually use free energy to generate their own motion and forces. Through interactions, active particles spontaneously organize in emergent large-scale structures with a rich range of materials properties. The active-matter paradigm is applied to living and nonliving systems over a vast dynamic range, from the organization of subnuclear structures in the cell to collective motion at the human scale. The diverse phenomena exhibited by these systems all stem from the defining property of active matter as an assembly of components that individually and dissipatively break time-reversal symmetry. This article outlines a selection of current and emerging directions in active matter research. It aims at providing a pedagogical and forward-looking introduction for researchers new to the field and a road map of open challenges and future directions that may appeal to those established in the area.
\end{abstract}

DOI: 10.1103/PhysRevX.12.010501

Subject Areas: Soft Matter

\section{INTRODUCTION}

\section{A. A bit of history}

In 1995, the Hungarian physicist Tamás Vicsek proposed a minimal model of bird flocking inspired by the physics of magnetism [1]. He showed that a collection of flying spins - self-propelled point particles traveling with fixed speed in a direction updated by alignment with neighbors in a noisy environment — can undergo a phase transition from a disordered state where the spins fly randomly in all directions to an ordered state of collective motion. Just a few months later, Vicsek presented his work in a seminar at IBM Yorktown Heights. John Toner and Yuhai Tu realized that they could turn Vicsek's agent-based model into a field theory and formulated what are now known as the Toner-Tu equations of flocking [2].

Truth be told, what physicists now know as the Vicsek model had effectively been previously formulated by Craig Reynolds, a computer scientist working for the animation industry, who in 1986 created Boids [3] —an agent-based simulation of collective motion that he employed, for instance, to generate the animation of flying bats in the

Published by the American Physical Society under the terms of the Creative Commons Attribution 4.0 International license. Further distribution of this work must maintain attribution to the author(s) and the published article's title, journal citation, and DOI.
1992 feature film Batman Returns. Indeed, the model appears even earlier in the literature, in theories of fish schools by Aoki [4] and Partridge [5]. While these contributions remained unnoticed by the physics community for some years, Reynolds, a leader in the development of three-dimensional animation, was awarded a Scientific and Technical Award by the Academy of Motion Picture Arts and Sciences in 1998. In the intervening years, the notion introduced by Vicsek that the collective dynamics of self-driven entities can be described as a nonequilibrium phase transition gained enormous popularity among physicists and was shown to provide a powerful framework for describing spontaneous organization on many scales. For their key contribution to the creation of the field of active matter, Vicsek, Toner, and Tu received the Lars Onsager prize of the American Physical Society in 2020.

The first published use of the term active matter appears to be in Ref. [6]. Active membranes appear a little earlier in the physics literature $[7,8]$. The term "active" in reference to fuel-driven transport across a membrane, against a concentration gradient, is standard in biology [9]. Active stresses in a fluid medium suffused with sustained energy conversion make their first appearance in Ref. [10].

\section{B. What is active matter?}

Today, the name active matter refers to any collection of entities that individually use free energy to generate their own motion and forces $[11,12]$. Through interactions, these 
active particles spontaneously organize in emergent largescale structures with a rich range of materials properties. The defining property of an active system is that the energy input that maintains the system out of equilibrium, whether truly internal or created by contact with a proximate surface, acts individually and independently on each "active particle." Hence, once the chemomechanical processes that convert fuel into motion are integrated out, the dynamics of such active entities breaks time-reversal symmetry (TRS) in a local and sustained matter. This should be contrasted with more conventional nonequilibrium systems that are displaced from equilibrium globally by an external force that picks out a direction in space, as in sedimentation under gravity, or are forced at the boundaries, such as through an imposed mechanical shear. Because of the breaking of TRS at the microscale, active systems do not obey detailed balance and can generate selfsustained flows and cyclical currents. Thus, steady-state movies of active dynamics run forward and backward do not look the same, as they would in Newtonian mechanics. Equilibrium statistical physics, which is founded on the notion of TRS and detailed balance, has played a fundamental role in the development of a predictive theory of passive matter. Active matter poses a fundamental problem in nonequilibrium statistical mechanics: What laws govern order, phase transitions, and fluctuations in systems in which the very particles constituting the system break detailed balance?

\section{Why is active matter important?}

Examples of large-scale self-organization in active matter abound in the living world [13], from the coordinated motion of cells in wound healing [14] to bird flocks [15] and human crowds [16,17]. Over the past 20 years, a number of synthetic analogs have also been engineered. These systems exploit energy-conversion avenues, ranging from the interplay of vibration and static friction [18-20] to a variety of autophoretic processes [21] [22], to endow particles from colloidal to macroscopic scales with selfpropulsion, often tunable through suitably applied light or electric fields [23]. Active matter spans an enormous range of scales, with realizations found inside the cell nucleus and in flocks of hundreds of thousands of starlings. It embodies a unique property of living systems, which is the ability to convert energy injected at the molecular scale into organized motion and function at the macroscopic scale. Research in active matter aims at understanding this defining property and at transferring this fundamental understanding to other disciplines. The importance of the field stems, therefore, from both the deep intellectual challenge of developing a predictive theory of the hierarchical organization ubiquitous in living systems and the potential for applying this understanding to the design of new bioinspired materials.
Over the past two decades, continuum and particle-scale theories, simulations, and experiments have uncovered striking properties of active particles that require conceptually new approaches and have brought key questions into sharper focus. Yet the field remains at the forefront of current research in soft matter, biological physics, and statistical physics, with high levels of activity and excitement.

\section{Outline}

The rest of the article is organized as follows. In Sec. II, we describe recent developments and future directions in understanding the self-sustained dynamics of orientationally ordered active fluids (Sec. II A). We focus on systems with nematic order and on the ubiquitous phenomenon of active turbulence (Sec. II B) and highlight the dynamics of topological defects in the nematic texture which spontaneously proliferate in the turbulent state (Sec. II C). An important open direction here is investigating the possible biological relevance of defects in organ and organism development and collective cell migration. In Sec. III, we focus on applications of active matter that may be achieved via the control of active flows (Sec. III A) and by learning to direct active assembly (Sec. III B). A challenge here is developing predictive theories of active assembly, with the ultimate goal of mimicking nature to construct machines made of smaller machines and capable of performing specified functions. To achieve this, it is important to understand the thermodynamics of active matter. This is the topic of Sec. IV, which focuses on the rapidly evolving use of ideas from stochastic thermodynamics to quantify deviations from equilibrium and formulate the nonequilibrium thermodynamics of active matter. Section V describes two new areas that have emerged in the study of active systems that seemingly break familiar symmetries of physical laws. In Sec. VA, we focus on the collective behavior of entities with effective interactions that are nonreciprocal, i.e., do not obey Newton's third law. This raises important and deep questions intimately related to the role of chirality (Sec. V B) and with surprising connections to non-Hermitian quantum mechanics. In Sec. VI, we highlight the role of active matter physics in developing a framework for describing the mechanical properties of cell collectives and biological tissue and conclude in Sec. VII, highlighting the breadth and connectivity of the field.

\section{ACTIVE FLOWS, BROKEN SYMMETRIES, AND TOPOLOGICAL DEFECTS}

Liquids can be made to flow by applying external forces at their boundaries. Collections of self-driven entities form active fluids that flow spontaneously with no externally applied forces or pressure gradients [24,25]. Since individual active units are often elongated or polarized along an axis, such self-driven fluids can exhibit liquid-crystalline 
order [12]. Order, in turn, couples to spontaneous flows, resulting in unusual rheological properties such as behavior similar to that of superfluids, excitability, and chaotic spatiotemporal dynamics known as bacterial or active turbulence. An important goal is to understand and classify dynamical phase transitions in these active fluids and the properties of various phases in terms of the symmetry of orientational order and the mechanisms that control energy dissipation.

This section provides an introduction to active matter and its general properties: spontaneous flow, active turbulence, and topological defects. These are relatively wellestablished research areas where important theoretical advances have been made over the past two decades, yet key challenges remain. In particular, the ubiquitous observation of self-sustained spatiotemporal chaotic dynamics in both engineered and living systems begs the question: What are the universal properties of active turbulence? Can we identify and classify various scenarios that drive the transition from spontaneous laminar flows to chaotic ones? Are the metrics used to quantify high-Reynolds-number turbulence useful in the active context?

\section{A. Polar and nematic active fluids}

Active particles with a head and a tail, such as bacteria [26], birds [2,15], polar vibrated grains [20], and Quincke rotors [27], can organize into states with polar (ferromagnetic) order and macroscopic mean motion-a flock. Apolar active particles, in contrast, form active fluids with nematic liquid-crystalline order, where the rodlike entities have a common orientation but no preferred direction. This more subtle type of orientational order, familiar from the physics of passive liquid crystals [28], is observed in active systems on many scales, from subcellular structures, such as the mitotic spindle that controls cell division, to suspensions of cytoskeletal filaments and associated motor proteins $[29,30]$, crawling bacteria [31-33], epithelia [34-36], monolayers of vibrated granular rods [18], and even entire multicellular organisms [37]. Active nematics have no net mean flow but display a complex self-sustained spatiotemporal dynamics that resembles the streaming used by cells to continuously circulate their fluid content. We note that scalar active matter described by a conserved concentration field coupled to a momentum-conserving fluid is also known to exhibit self-driven flows [38,39], but this is beyond the scope of the present article.

The uniform ordered bulk states of both polar and nematic active fluids are generically unstable, at any activity [24], to self-sustained chaotic spatiotemporal flows or active turbulence [40], as exemplified in Fig. 1. The vortical flows look qualitatively similar in both types of system, raising the question of how the symmetry of the orientational order affects the behavior and scaling properties.
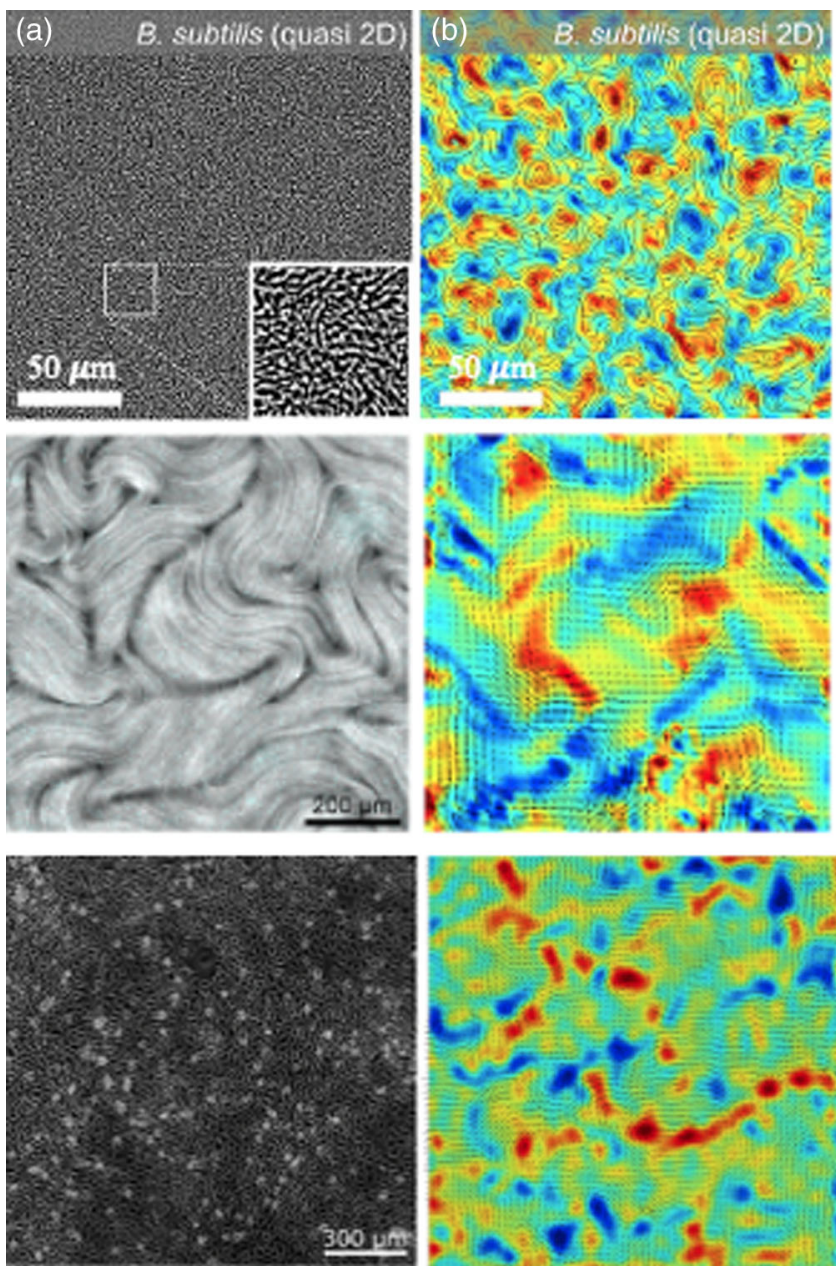

FIG. 1. Experimental realizations of active turbulence, from top to bottom: a layer of Bacillus subtilis (adapted from Ref. [26]), a suspension of kinesin-powered microtubule bundles (courtesy of Linnea Lemma and Zvonimir Dogic), and a layer of MDCK cells (adapted from Ref. [36]). The left column shows images of the system, while the right column displays snapshots of the local vorticity.

The order parameter for the flocking transition is the mean polarization vector or self-propulsive velocity of the active entities, which, of course, has the same vectorial symmetry as the flow velocity of the fluid. One can then often describe the dynamics in terms of a single vector field through the Toner-Tu equations [41] which marry the Navier-Stokes hydrodynamics of simple fluids to the relaxational dynamics of magnets.

Because of the apolar nature of the ordered state, the nematic order parameter is a tensor field $Q_{i j}=\left\langle\sum_{\alpha} \nu_{i}^{\alpha} \nu_{j}^{\alpha}-\right.$ $\left.\delta_{i j} / d\right\rangle=S\left(n_{i} n_{j}-\delta_{i j} / d\right)$ that measures the uniaxial anisotropy of the distribution of the orientation $\nu^{\alpha}$ of individual molecules, with $d$ the system's dimensionality. The nematic is a fluid with a finite value of $S$ and spontaneously broken orientational symmetry along the director $\mathbf{n}$.

In the regime appropriate to most active fluids where inertia is negligible, and assuming constant density, the 
dynamics of an active nematic is then described by the interplay of relaxation and flow-induced reorientation of the $\mathbf{Q}$ tensor and force balance encoded in the Stokes equation for the flow velocity $\mathbf{v}$ :

$$
\eta \nabla^{2} \mathbf{v}-\nabla p+\nabla \cdot \sigma^{a}=0
$$

where we for simplicity neglect stresses due to liquidcrystalline elasticity. The pressure $p$ is determined by requiring incompressibility: $\boldsymbol{\nabla} \cdot \mathbf{v}=0$. The active stress $\boldsymbol{\sigma}^{a}=\alpha \mathbf{Q}$ describes the dipolar forces exerted by the active nematogens of the surrounding fluid [25,42]. It is controlled by the activity $\alpha$ that incorporates the biomolecular parameters (such as the concentration of motor protein and of ATP) that drive active motions. The sign of $\alpha$ depends on whether active forces are extensile $(\alpha<0)$, as in microtubule nematics, or contractile $(\alpha>0)$, as may, for instance, be the case for certain actomyosin networks. In active nematics, the instability of the ordered state arises from the competition between energy input from active stress that drives disorienting flows and orientational relaxation that restores alignment [43]. Because of the feedback between flow and orientational distortions, vortical flows are accompanied by proliferation of topological defects in the nematic texture.

\section{B. Active turbulence}

The chaotic spatiotemporal dynamics known as active turbulence is a phenomenon distinct from the well-known inertial turbulence of high-Reynolds-number fluids, perhaps more akin to the elastic turbulence observed 20 years ago in sheared polymer solutions [44], where nonlinear elastic effects can destabilize laminar flow. The distinctive feature of active or bacterial turbulence is that the system is destabilized by stresses generated internally at short scales and then self-organized to larger scales through the interplay of interactions and dissipation. As in inertial turbulence, a useful quantity to characterize velocity correlations is the spectrum of kinetic energy and of vorticity of the flow, with the latter providing direct information about the distribution of vortex sizes. At intermediate scales, the turbulent flows of inertial turbulence are self-similar, resulting in the characteristic Kolmogorov scaling of the kinetic energy spectrum [45]. In active turbulence, in contrast, the spectrum of energy injection that drives the chaotic flows is not externally imposed but rather selforganized. This essentially arrests the energy cascade and leads to the selection of a length scale $\ell_{a}=\sqrt{K /|\alpha|}$ determined by the balance of active and elastic stresses (of strength determined by the nematic stiffness $K$ ) that controls both the spacing of topological defects in the nematic texture and the size of the flow vortices [46]. As a result, these chaotic flows can be equivalently characterized by examining the statistics of flow vortices or that of the nematic disclinations. Numerical studies have established the emergence of power laws in the energy spectrum and other scalings analogous to, but different from, Kolmogorov's scaling of inertial turbulence [40]. A remarkable result is the demonstration of the "universality" of the scaling properties of active nematic flows and associated defects in the nematic texture across systems and scales [46]. An open direction is the search for multiscaling identified by examining higher-order velocity correlations, as done in inertial turbulence [47]. The study of turbulence using metrics from the theory of dynamical systems, such as Lyapunov exponents or topological entropy, is in its infancy. More work is needed to see if this is a useful approach. For a more extensive discussion of active turbulence, we point the reader to a recent critical review of active turbulence [40].

\section{Order parameters and inertia}

An important open question is quantifying the difference between chaotic active flows in polar and nematic fluids. For instance, in polar fluids, nonlinear terms describing self-advection of the order parameter seem to be important in driving energy transfer across scales. How does this compare to the role of self-advection in inertial turbulence? In contrast, there is no self-advection in active nematics, and advective nonlinearities seem to play a secondary role relative to flow alignment and flow-induced rotation of the order parameter in driving nonlinear flows. Is this difference then important for explaining the vortex statistics in the two systems?

It has also recently [48] become clear that the inclusion of inertia transforms our understanding of extensile or "pusher" polar active suspensions. A new dimensionless control parameter $R \equiv \rho v_{0}^{2} / 2 \sigma_{0}$, suggestive of an effective Reynolds number, emerges, where $\rho$ is the total mass density, $v_{0}$ the self-propulsion speed, and $\sigma_{0}$ the characteristic scale of active stress. Speed matters, and there are two in the problem. The instability advances with a finite velocity $\left(\sigma_{0} / \rho\right)^{1 / 2}$ in a treatment in which inertia $(\rho)$ is taken into account, and, if $v_{0}$ is large enough, the suspension can outrun and, thus, eliminate the instability. The inescapable Stokesian instability of flocks in a fluid is simply the $R=0$ limit of a rich phase diagram, including a flocking transition driven by motility, separating a phaseturbulent but ordered flock from a defect-turbulent statistically isotropic state [48].

\section{Role of dissipation and "order from disorder"}

Another open challenge is understanding the role of different dissipative processes and associated length scales in mediating the onset of nonequilibrium dynamical steady states. In many experimental realizations, dissipation is controlled by the combination of viscous stresses and frictional drag with an external inert medium, described by an additional force density $-\Gamma \mathbf{v}$ on the left-hand side in Eq. (1). This introduces another length scale in the 
problem, the viscous screening length $\ell_{v}=\sqrt{\eta / \Gamma}$. Numerical work indicates that the interplay between $\ell_{v}$, the active length $\ell_{a}$, and the nematic coherence length may result in vortex lattices, reminiscent of those familiar in type-II superconductors and Bose-Einstein condensates $[49,50]$. More work is needed, however, to understand how the interplay between these two dissipation mechanisms may mediate the emergence of such order from disorder.

\section{Active nematics in three dimensions (3D)}

These systems are only beginning to be explored. Experimental realizations of 3D microtubule nematics show that in these systems turbulent flows are accompanied by the formation of neutral defect loops with zero topological charge. No charged 3D defects have been observed [51]. The structure of the topological defects generated by active flows in $3 \mathrm{D}$ and especially the connection between defects and flow structure remains to be explored. Recent numerical work also shows that, in 3D active fluids, spontaneous breaking of chiral symmetry leads to parityviolating Beltrami flows $[52,53]$. This points to the need to explore the role of chirality in active flows, in both 3D and 2D [54].

\section{Linking to interfacial instabilities}

Intriguing connections are also apparent between active mixtures and turbulent multiphase flows, where the interfaces between the coexisting phases play an important role in controlling the dynamics. Similarly, the largely unexplored interfacial properties of mixtures of active liquid crystals and passive fluids should control the highly nonequilibrium dynamics of these systems, with possible relevance to liquid-liquid phase separation in biological contexts [55]. Worth exploring are connections between active and passive interfaces and the interfaces found in staircases in density-stratified fluids [56] or in magnetized plasmas.

\section{Need for experiments}

Finally, even if theorists succeed at establishing classes of active turbulence distinguished by different scenarios of paths to chaotic flows and critical exponents, we still need quantitative experimental tests of these predictions. This requires measurements of energy spectra in controlled settings, most likely to be carried out in synthetic active analogs or in vitro systems. An important question, of course, is whether active turbulence plays a role in biology. Active stress fluctuations that in active nematics generate spontaneous flows, whether turbulent or orderly, may, for instance, help overcome a slow passive diffusion process and promote mixing and transport in the cell cytoplasm [57,58], but the connection needs to be made more quantitative.

\section{Topological defects}

Topological defects are widely explored in both active and passive systems. What are the new features of active defects and the challenges ahead? In active fluids, spontaneous local currents turn the defects themselves into selfpropelled entities. The interacting defect gas then forms an active fluid with the possibility of dynamically generated order of the defect themselves, such as polar (ferromagnetic) order on a larger scale than the microscopic nematogens, and nonreciprocal interactions between defects. Furthermore, the spontaneous pair creation of defects driven by activity means we need a full many-body theory to describe the interacting defect gas-this does not currently exist.

\section{Topological defects as a Coulomb gas in $2 D$ passive matter}

To fill in with some background, topological defects are zeros of the order parameter field classified by their topological charge (see Fig. 2 for a definition). In passive systems, defects play a fundamental role in many twodimensional phase transitions, the poster child being the Berezinskii-Kosterlitz-Thouless (BKT) transition [28,59]. First of all, the symmetry breaking in the BKT transition is described by a subtle topological order in the defects themselves [in this case, $X Y$ or U(1) vortices] rather than the spontaneous breaking of a global symmetry. At high temperature vortices are randomly distributed, whereas below a critical temperature they form measurable vortex-antivortex bound pairs. The transition is then described as vortex unbinding. Second, the defect degrees of freedom are the slowest, or rate-limiting, degrees of freedom in the approach to the ordered phase. The BKT transition is, therefore, fundamentally formulated as a many-body theory of a gas of defects by mapping the defects onto interacting Coulomb charges. Similarly, the half-integer strength disclinations of two-dimensional nematics control the development of nematic order in the isotropic to nematic $(I-N)$ phase transition [60].

\section{Active defects as a gas of self-propelled Coulomb charges}

For active nematics, a striking new phenomenon emerges. Defect-antidefect pairs are spontaneously created and annihilated [29]. Furthermore, the elementary $+1 / 2$ strength disclinations become self-propelled [18]. When hydrodynamic flows are included, self-propulsion originates by virtue of a monopole moment of velocity at the defect cores arising from the induced dipolar fluid flow determined by the nematic texture of the defect $[62,63]$ (Fig. 2). The $+1 / 2$ defect has a twofold-symmetric cometlike pattern with a head and a tail that define a local polarization vector. We stress that the self-propulsion of the $+1 / 2$ defect emerges spontaneously as a direct 


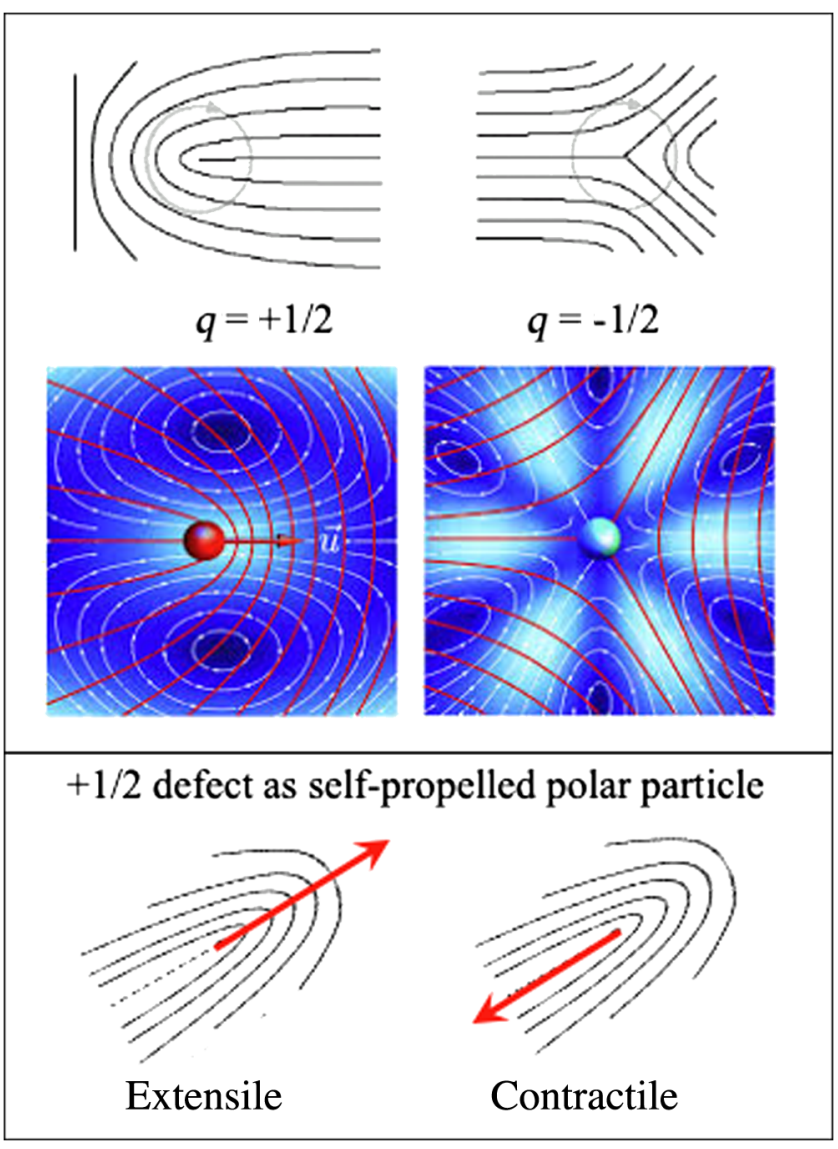

FIG. 2. Topological defects in active nematic liquid crystals. Top and middle rows: configuration of the director texture for a $+1 / 2$ (left) and a $-1 / 2$ (right) defect (top row) and associated flow fields (middle row). The topological charge $q$ is defined as the net rotation (in units of $2 \pi$ ) of the order parameter as one encircles the defect. In the left frame, the director rotates by $\pi$ in the same direction as the path is traversed, yielding $q=+1 / 2$. In the right frame, the director rotates by $\pi$ in the opposite direction as the path is traversed; hence, $q=-1 / 2$. The magnitude of the flow field around an isolated defect is shown in blue, with the white lines representing the streamlines of the flow (adapted from Ref. [61]). The red lines are tangent to the director field. Bottom row: $+1 / 2$ defects as self-propelled polar particles. The red arrow shows the self-propulsion speed of $a+1 / 2$ defect powered by flows induced by extensile (left) and contractile (right) active stresses.

consequence of the simultaneous breakdown of TRS due to active stresses and spatial symmetry due to the defect's own polar structure. The $-1 / 2$ strength antidefect, in contrast, is not directly self-propelled by virtue of a threefold symmetry in its nematic texture and resulting vanishing fluid velocity at the defect core. The value of the self-propulsion velocity of $\mathrm{a}+1 / 2$ defect is proportional to the activity, at least perturbatively.

There has been progress in describing active nematic flows in terms of the dynamics of the topological defects in the nematic texture by developing a description of defects as an interacting gas of active quasiparticles [64].
The self-propulsion of the $+1 / 2$ defect can oppose Coulomb attraction mediated by nematic elasticity. For sufficiently high activity, a defect pair can even dynamically unbind, in an active version of the BKT transition [64]. The existence of a finite activity threshold for the active BKT unbinding may seem surprising. One might imagine that directed self-propulsion of the $+1 / 2$ defect can always overcome attraction and there is no active nematic phase at all. A closer look, though, shows that defects do not separate ballistically. Noise-induced torques lead to rotational diffusion and the stabilization of an active nematic phase.

Existing work does not, however, tackle the important question of how defect pairs are unbound by active stresses. Answering this question necessitates a microscopic calculation of the rate of defect pair creation and annihilation as a function of activity and is, so far, beyond reach. A complete theory of the active defect gas, in fact, requires a manybody formulation that accounts for defect creation, just as electron-positron pair creation requires the formalism of quantum electrodynamics. It may also be that the polarization of defects is not an independent degree of freedom but instead emerges from the detailed dynamics of the set of locations of defects. Attempts to sort out this issue raise the important question of when and to what extent one can fully describe the system's dynamics by tracking the defect motions, without also following the dynamics of the nematic texture, which could lag behind that of the defects $[30,65]$.

\section{States of defect order}

Since the interacting defect gas is itself a mixture of active polar particles $(+1 / 2)$ and passive particles $(-1 / 2)$ with aligning torques, a natural question is whether active defects may organize in emergent ordered structures. Understanding the possible local and global order of defect arrays in the active nematic phase remains an open challenge. Numerical work suggests that defects and associated flow vortices may organize in ordered lattices when the screening length controlling the interplay between viscous and frictional dissipation is comparable to the defect spacing [49]. A hydrodynamic description of the defect gas shows that, when flow is slaved to texture deformations, the spontaneous breaking of rotational symmetry leads to a collectively moving defect-ordered polar liquid - a flocking state of defects [66]. This state emerges at activity levels beyond the turbulent state discussed earlier, a remarkable example of order from disorder. Polar order of $+1 / 2$ defects is observed in simulations of "dry" nematics [67] [68-70] but seems to occur at intermediate activity. Theoretical models also predict local nematic (i.e., antiparallel) order of the $+1 / 2$ defects [71,72], consistent with some recent experiments [71] but at odds with earlier work predicting [73] and observing large-scale nematic ordering [74]. Numerical solutions of 
continuum models also indicate the possibility of ordered defect lattices with intriguing analogies to superconductors [75]. It is clear that more work is needed to sort out these conflicting results. The proper treatment of incompressibility, most likely the experimentally relevant case for wet systems under planar confinement, remains a challenge in the modeling of defect dynamics.

\section{Defects in biology}

Topological defects are distinctive singularities in both the mathematical and physical sense and, as such, are natural places for functionalization or the generation of higher-level structures. The unique local environment of a defect is exploited in passive systems, using placeexchange chemical reactions in which molecules bind directly and preferentially at defect sites [76]. Similarly, biological structures have long been known to functionalize defects. A well-known, perhaps passive, example is the common Adenovirus40 (Ad40) which has one fibril attached to each of the topologically minimal 12 pentons (pentagonal-shaped regions formed by proteins) of its viral capsid, creating 12 arms with which to latch on to target cells [77].

Examples of the role of defects in active biological systems are now emerging at a rapidly growing pace. There are experimental demonstrations of the biological function of topological defects as loci of cell extrusion in cell layers $[34,35,78]$, seeds of multilayer formation in dense bacterial sheets [79,80] and mammalian cells [81], and organizing centers of morphogenetic processes in multicellular organisms [37].

Dense monolayers of spindle-shaped neural progenitor cells (NPCs) exhibit distinct nematic order with a finite concentration of $\pm 1 / 2$ defects. Nematic order is less pronounced in epithelial Madin-Darby canine kidney (MDCK) cell layers, but defects can also be tracked there. In NPC layers, cells accumulate at $+1 / 2$ defects, leading to the formation of three-dimensional mounds-a beautiful example of a nontrivial actively generated structure nucleated by a topological defect [34]. At the $-1 / 2$ defects, in contrast, cells are depleted. It is suggested that an interplay between anisotropic friction and active stress is responsible for this source-sink behavior, but much remains to be clarified. In MDCK epithelia, cells are vertically extruded at $+1 / 2$ defects and die-a process known as apoptotic cell extrusion [35]. Cell extrusion here seems to be driven not by increased cell density (cell crowding) but rather by the mechanical stresses associated with the texture of a defect. That such a basic biological process as cell death is intertwined with the presence and formation of active nematic defects is both striking and powerful and could well have widespread applicability.

Recent developments also highlight the role of nematic defects in the formation of multilayer structures in both bacteria $[79,80]$ and NPCs [81]. Especially intriguing is the suggestion put forward in Ref. [81] that the structure of nematic defects and the nature of active forces may together control the relative cell orientation in multilayered cell structures. Most of these experimental findings are at best qualitatively understood and await theoretical input.

More recently, it has been shown that in regenerating Hydra, supracellular actin fibers also lead to active nematic order with significant morphological features developing at long-lived but dynamic +1 defects [37]. Here, the topology of the tissue is spherical, and so, in the absence of topology change, the nematic texture is required to have a net topological charge of 2 fixed by the Euler characteristic of the sphere. This requirement has minimal solutions consisting of either four $+1 / 2$ defects, two $+1 / 2$ 's and one +1 , or two +1 's. Any additional number of defectantidefect pairs also satisfy the constraint. In Hydra, two +1 defects form and nucleate the head and tail. This is interesting, because the energy associated with defect formation goes as the square of the defect charge; hence, the formation of $a+1$ defect is energetically disfavoredthe intrinsic elastic energy of a +1 defect is twice that of two $+1 / 2$ 's. Interactions between active $+1 / 2$ defects, however, lead to attractive forces that lead to tightly bound states of two $+1 / 2$ defects [64], and this state has the same far-field texture as a +1 defect. Thus, like-sign defectdefect attraction in active nematics is responsible for the head and tail of regenerating Hydra - amazing. Once again, the distinctive environment in the neighborhood of an essential singularity drives fundamental morphological development. The quantitative exploration of the role of defects in morphogenesis is only just starting [82]. For instance, in the budding of tentacles in Hydra, do changes in the morphology of the actin supracellular structure with associated defect formation precede or follow cell accumulation at the budding site? And are there morphogenetic signaling proteins that drive cell accumulation? Further work coupling theory and controlled experiments is needed to elucidate the interplay between structure, biochemical signaling, mechanics, and curvature in controlling these processes.

Finally, seemingly turbulent dynamics of scalar chemical fields associated with wave propagation patterns of signaling proteins was recently observed in the membrane of starfish egg cells [83,84]. The observed "spiral defect chaos" resembles spiral-wave patterns in reaction-diffusion systems and in the heart, but the observation of such dynamics in vivo and the identification of its role in biochemical signaling suggest the importance of biochemical patterns in controlling multicellular organization and tissue mechanics in biological development. Understanding the statistics of such patterns in living systems and connecting them to the propagation of mechanical forces is an important area for exploration. 


\section{ENGINEERING ACTIVE MATERIALS}

Living active matter has inspired the creation of synthetic materials composed of energy-dissipating components either assembled from cell extracts or engineered as microswimmers driven by a variety of phoretic mechanisms. These systems allow controlled experiments that have played a key role in guiding and testing active-matter theory. We are now on the verge of achieving control of active matter, opening up a route toward engineering new materials with lifelike functionalities.

\section{A. Harnessing active flows}

Passive liquid crystals have enormous technological applications thanks to a detailed understanding of the role of boundary conditions and the response to external fields that allows remarkable control of these materials. The dream is to achieve equal control of active fluids so that self-organized active flows can be utilized, for instance, for microfluidic transport and tissue engineering or for powering machines at the micro- and nanoscale. Can we then achieve robust behaviors by controlling topology and geometry of active flows? While we know that chaotic active flows can be tamed into stable laminar flows through geometry and confinement $[85,86]$, we need to learn how to shape boundaries and vary boundary conditions to achieve specific control of flow in the bulk. Applications require a quantitative understanding of anchoring behavior, wetting and interfacial properties of active liquid crystals, and their response to external fields.

\section{Controlling flows with boundaries and geometry}

While geometric confinement is relatively well explored, more recently it has been shown that spatial changes in Gaussian curvatures can regulate specific defect structures and direct active flows [87-91]. The group of Sagués has also demonstrated experimentally the possibility of achieving magnetic control of active liquid crystals by interfacing them with passive ones [92] (see Fig. 3). The control of active fluids through patterned substrates is a promising direction where much remains to be explored both experimentally and theoretically.

\section{Controlling flows by patterning activity}

An ambitious challenge is designing flows that cannot be achieved by control of external boundaries. Progress in this direction has been achieved by engineering active suspensions of cytoskeletal filaments cross-linked by optogenetically modified proteins, where the activity can be turned on and off with light illumination. This allows the creation of controlled spatial patterns of active and passive regions within a given sample and the temporal reconfiguration of such patterns with suitable light pulses [93-96]. On the theoretical side, we are faced with the task of formulating a quantitative framework that will allow us to predict how we

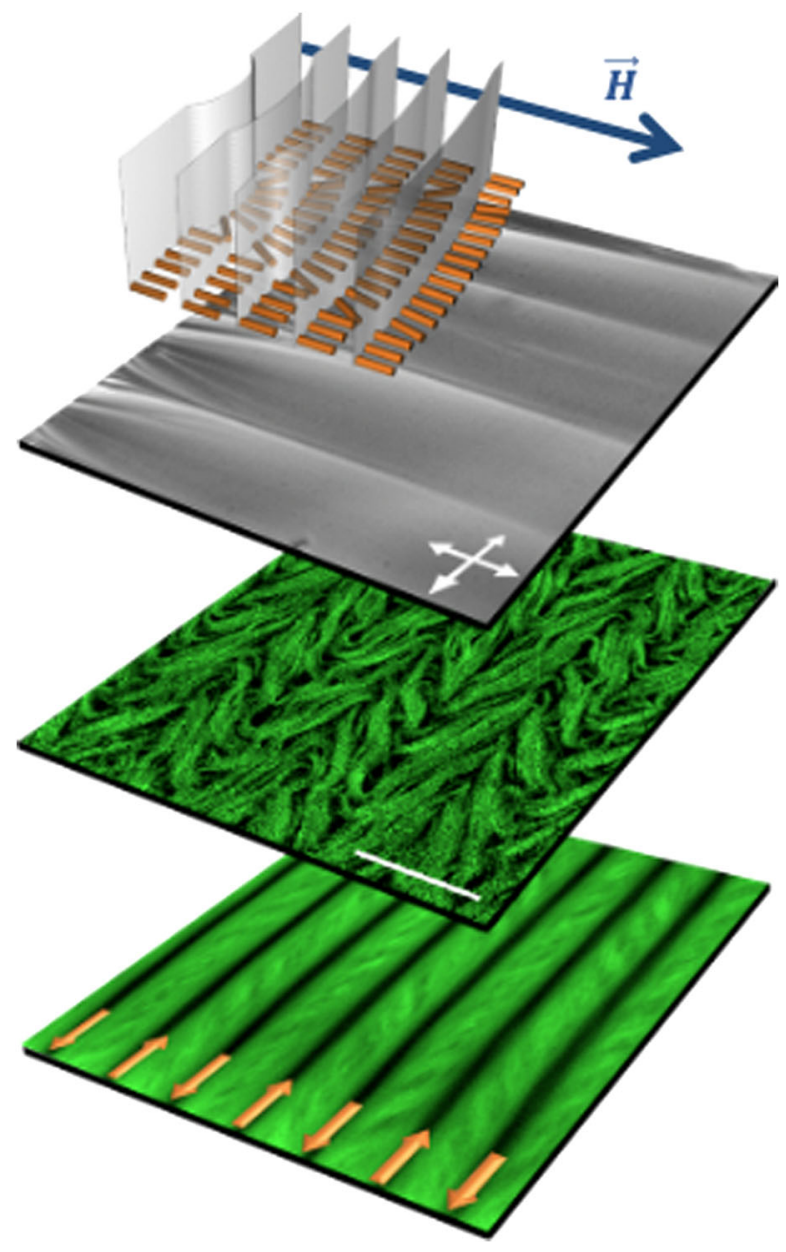

FIG. 3. Aligning an active nematic with a magnetic field by interfacing it with a passive nematic layer. The figure, adapted from Ref. [92], shows, from top to bottom, the configuration and an optical micrograph of the underlying passive liquid crystal aligned in the smectic A (SmA) phase by application of a magnetic field (top); a snapshot of the active nematic obtained by fluorescent confocal microscopy showing the alignment in "kink walls" (also referred to "arches" in the literature) induced by the coupling to the SmA structure of the passive layer, and the time average of the dynamical pattern in the active nematic layer, with arrows indicating antiparallel flow directions (scale bar, $100 \mu \mathrm{m})$.

must sculpt activity in space and time to design specific active flows. This requires developing theoretical and experimental tools to locally map out and quantify active stresses and connect stress to structure and flow. Given active flows are directly coupled to topological defects in the orientational order, a complementary strategy focuses on using spatially inhomogeneous activity to confine and guide defects. Recent theoretical and experimental work demonstrates that activity gradients engender pressure gradients that effectively act like electric fields on topological charge $[66,96]$. Further work is needed to understand how far the effect of activity extends in both length and time into the passive regions to achieve the design of 
emergent defect and flow states that exhibit both spatial and temporal organization. The ultimate challenge is to build materials that mimic biology, where the behavior is controlled by processes that are entirely internal to the system. The next experimental step, therefore, is to design a system that can sense changes to flow patterns and respond to such changes.

\section{Other emerging strategies}

Viscoelasticity of the suspending medium may also provide a mechanism for controlling active flows $[97,98]$. Recent experimental and theoretical work, in fact, shows that viscoelasticity not only can calm chaotic flows [99-102], but can also be used to simultaneously tune spatial and temporal organization [103]. This is distinct from the well-studied organization of interacting oscillators, as it provides a mechanism for the spontaneous organization of dissipative entities with no internal clock into macroscopic emergent states exhibiting sustained oscillations.

Another intriguing direction is the use of active fluids for fluid-mediated computation strategies. The use of fluid networks for storing and transmitting information is exploited in certain organisms, such as the slime mold Physarum polycephalum which uses fluid networks to solve optimization problems, although the mechanisms through which it achieves this are yet to be understood [104]. Pressure-driven microfluidic circuits are employed to perform Boolean computation [105]. The design of microfluidic devices powered by active fluids and capable of performing logical operations by exploiting the interplay of internal drive and constraints imposed by incompressibility is suggested theoretically [106] but deserves further exploration.

\section{Role of theory}

Finally, to answer many of the questions raised above, it is necessary to carry out detailed coarse-graining to connect microscopic interactions to predictive descriptions at the macroscopic or continuum level. New approaches are being developed to translate large sets of experimental data on the microscopic dynamics of individual active entities into lower-dimensional models for the dynamics of a few coarse-grained fields. These range from mode decomposition approaches well tested in condensed matter and turbulence theory [107] to inferring complex interactions from the statistics of individual stochastic trajectories $[108,109]$. Recent work demonstrates the feasibility of using optimal control theory to calculate inputs capable of rapidly restructuring active nematic flow [110]. These approaches and the related application of optimal mass transport to identify the most efficient way of reconfiguring and transporting active fluids, for instance, in complex microfluidic devices, deserves much further exploration. A new frontier is the use of machine learning for inferring the parameters of continuum models directly from experimental data and even inferring the continuum equations of motion. The power of this approach is demonstrated for active microtubule nematics [111,112], bacteria [113,114], and active Brownian particles [115] but promises to be a fruitful tool for modeling other active systems.

\section{B. Directing active assembly}

Since the first catalytic microswimmer was engineered now almost 20 years ago [116] (see Fig. 4), a broad class of "machines" powered by light or chemical sources has been developed, including more sophisticated catalytic colloids, enzymes, metabolic networks, Marangoni droplets, and Quincke rotors [23]. In many of these, the swimming speed or degree of activity can be controlled with light or steered with external fields. Thanks to the unprecedented control achieved in experiments, these active colloids, as they are broadly called, provide an important testing ground for active-matter theory and offer the potential for a variety of technological and biomedical applications, from microscale stirrers to targeted drug delivery [117-119]. Fulfilling this potential, however, requires a quantitative understanding of the propulsion mechanisms of these active particles and of their interactions, as well as the development of theories that connect microscopic properties to emergent behavior. An important difficulty, as compared to passive assembly, is that while in equilibrium we generally know the equilibrium state, the steady state of active systems is typically not known. The lack of knowledge of possible target states impedes the development of a systematic procedure for guiding assembly.

\section{Emergent organization of active colloids}

Active colloids continuously draw energy from an ambient nonequilibrium medium, and their interactions are mediated by chemical phoresis and hydrodynamic flows $[23,120]$. As a result, the effective pair interactions

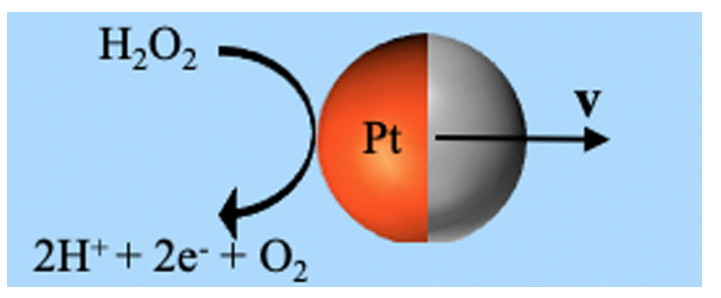

FIG. 4. The simplest example of an active colloid is a micronsize polystyrene bead half coated with platinum immersed in a hydrogen peroxide solution and powered by solvent concentration gradients that interact with the particle's surface. The platinum catalyzes the breakdown of $\mathrm{H}_{2} \mathrm{O}_{2}$ in oxygen and water, generating anisotropic concentration and charge currents that turn the micron-size bead into a swimmer. The speed of these synthetic swimmers is controlled by the concentration of $\mathrm{H}_{2} \mathrm{O}_{2}$ and is typically of order of tens of microns per second, comparable to that of flagellated bacteria like Escherichia coli. 
are generally nonreciprocal [121-124]. Such nonreciprocity is especially important in active colloidal mixtures. Its consequences can be fascinating and are discussed further below. Progress has been made in the classification of diffusiophoretic colloids by relating single-particle features and the symmetry and source of their pair interaction to the resulting variety of pair dynamics, such as bound dimers or orbiting pairs, versus scattering states [125]. An important open challenge is now establishing a quantitative connection between types of pair interactions and emergent behavior to formulate a classification of active colloids that relates their microscopic properties to their collective organization at a large scale and to map out the phase diagram of each class of particles. Tackling this challenge requires systematic theoretical work closely coupled to experiments to relate the parameters of theoretical models to experimental ones.

Some progress has been made in this direction (Fig. 5), but achieving a complete classification requires a better understanding of hydrodynamic interactions, that is, interactions mediated by fluid flow, especially their role in driving coordination of swimmer orientation. For instance, it is known that hydrodynamic interactions can hinder or arrest the motility-induced phase separation of scalar active matter [126]. More generally, do flow-mediated couplings promote or hinder structure formation? What is the role of hydrodynamic interactions relative to ones mediated by chemical fields? Can we even quantitatively distinguish the two? A quantitative understanding of the role of these competing mechanisms is necessary in order to learn how

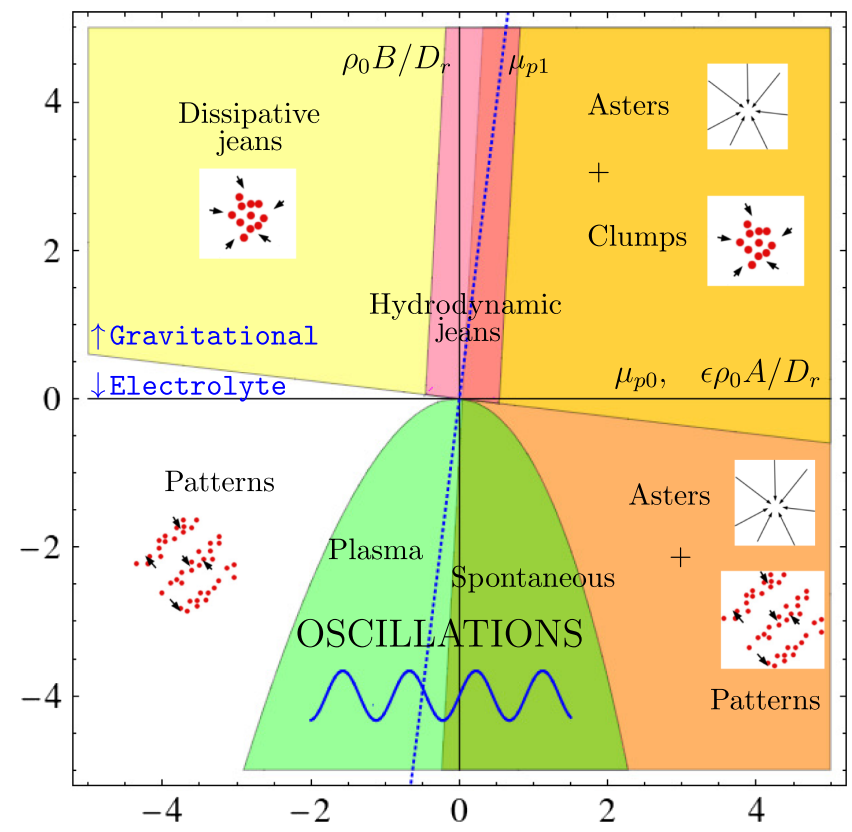

FIG. 5. Nonequilibrium phase diagram of polar active colloids in the plane spanned by adimensionalized "chemotactic" reorienting response coefficient $A$ (horizontal axis) and phoretic motility $B$ (vertical axis). Reproduced from Ref. [133]. to direct the assembly of such active particles to build smart and reconfigurable materials. A complementary approach, inspired by successes in metamaterial design, is to tackle the inverse problem of configuring an active system and active interactions to obtain specific target structures or target flows or to perform specific functions [127-129]. This approach is still in its infancy in the context of active matter, although progress is being made by local tuning of physical interactions [102], via machine-learning methods [112], and through the recently proposed notion of biased ensembles [130-132]. This type of work may provide connections with methods developed in the robotics community.

\section{Retardation and memory effects}

Most theoretical work so far assumes that the dynamics of the colloidal particles is much slower than the timescale for diffusion of the chemical fuels that power it. In this limit, the effective interactions generated by chemical fields (both self-interactions that drive the particle's motion and interparticle interactions) can be treated as instantaneous. Some work considers the opposite limit of very slow chemical diffusion [134], but the intermediate situation likely to be relevant to many diffusiophoretic colloids where these timescales are comparable has not been addressed. Intermediate timescales of chemical diffusion can result in time-delayed interactions and memory effects, which may engender new emergent behavior, such as traveling and oscillating states. A similar role is known to be played by viscoelasticity of the ambient medium. In both cases, these effects act like an "effective inertia" on the dynamics, which could mediate emergent structures that exhibit both spatial and temporal organization, as shown recently for bacteria swimming in a viscoelastic fluid [103].

\section{Emergent organization from biochemical control}

It is also tempting to draw an analogy between the chemically driven dynamics of active colloids and biochemical patterns [83]], as well as spontaneously assembled structures resembling complex molecules in a variety of active orientationally ordered systems [135,136]. Biochemical patterns are ubiquitous in biology, and they control organization at both the subcellular and the multicellular levels $[137,138]$. Spatiotemporal symmetry-breaking transitions in biochemical patterns are essential in triggering morphological changes during development, at both the unicellular and multicellular level. The realization of cell and tissue-scale deformations is achieved through intracellular force networks that translate localized biochemical signals into effective mechanical stresses that determine the global shape dynamics. Biochemical regulation is studied extensively in the context of nonlinear chemical reaction networks [139]. Biochemically driven organization is also demonstrated experimentally through the ability to tailor density patterns in bacteria swarms with 
specific illuminations $[140,141]$. It would be interesting to explore what biochemical and biophysical pattern formation, regulation, and mechanochemical feedback mechanisms can teach us about active organization [142]. The long-term goal is to learn to regulate phoretic effects to control pattern formation in synthetic active systems. The creation of polar flocks or apolar active liquid crystals made from anisotropic self-phoretic colloids is an interesting challenge.

Finally, recent experiments suggest the possibility of building active colloidal systems controlled by real-time feedback [143-145]. This is a first step toward the design of responsive materials capable of sensing their environment and responding in organized and prescribed ways. This work connects directly with advances made in the robotics community toward building smart flocks that can sense their environment [146] and adapt to it and has implications for the understanding of information flow [147-149].

\section{THERMODYNAMICS OF ACTIVE MATTER}

Life's ability to exploit energy across scales is remarkable. Living systems, operating far from equilibrium, can harness energy at the molecular scale through ATP hydrolysis and dissipate it on much larger spatiotemporal scales. Energy dissipation results in emergence of selforganized structures that span the entire length of a cell, such as the actomyosin cortex $[150,151]$ or mitotic spindle $[152,153]$. On the scale of populations, suspensions of bacteria, cells in tissues, and flocks of birds can form remarkable swirling patterns, due to their nonequilibrium dynamics. These examples and all active-matter systems, in general, are "open" from the thermodynamic point of view. How do information, energy, and entropy flow and transform due to interactions with the system's environment or within the system itself? Efforts toward a fundamental understanding of the physical and information-theoretic dynamics of these systems, and its exploitation to discover novel design principles, are another exciting frontier in active matter.

Current efforts are importantly focused on answering two questions: How far from equilibrium are active-matter systems, and what can we do far from equilibrium? Beyond this, can we push beyond quantification of distance from equilibrium and find new ways to exploit time-varying interactions, fluctuations, and phase-space structures to generate useful work [154] and design innovative active engines [155-157]? Other recent efforts focus on exploring what types of steady states are efficiently assembled through engineered dissipation [131]. How does dissipation result in self-organization and maintenance of spatiotemporal patterns in active matter [158]? Can we control activity to enable optimal nonequilibrium environmental energetic and information transfer? Does dissipation engineering enable programmable active matter? This is a first step toward developing thermodynamically efficient methods for actively modulating the phase-space structure of active-matter systems to enable adaptive control and learning.

\section{A. How far from equilibrium is active matter?}

Work on nonequilibrium thermodynamics has had major successes in building the thermodynamics of far-fromequilibrium systems, with universal fluctuation theorems and other model-free results that deeply constrain the probability distributions for quantities like applied or extracted work and entropy production [159]. In recent years, progress has been made in applying these concepts to active matter.

An important challenge is developing measures of dissipation and irreversibility that allow us to distinguish between active and passive systems, as well as to quantify the difference between active systems that are driven out of equilibrium by internal processes and systems driven out of equilibrium by externally applied forces and perturbations. Quantifying dissipation will open new avenues for probing self-organization principles in these far-from-equilibrium systems. Below, we introduce three frameworks from nonequilibrium statistical physics that are promising candidates for providing insight for this very exciting endeavor.

\section{B. Departure from fluctuation dissipation theorem}

A consequence of the time-reversal symmetry of equilibrium is the fluctuation-dissipation theorem (FDT). Put simply, for a small perturbation at frequency $\omega$ the systems response is linear and completely characterized by the generalized susceptibility $\chi^{\prime \prime}(\omega)$. Near equilibrium, timereversal symmetry relates this response to the intrinsic thermal fluctuations characterized by the power spectrum $S(\omega)$ (Fourier transform of a correlation function) through the FDT:

$$
S(\omega)=\frac{2 k_{B} T}{\omega} \chi^{\prime \prime}(\omega) .
$$

Here, $k_{B}$ is Boltzmann's constant, and $T$ is the temperature of the surroundings. A major consequence is in refining our understanding of the material coefficients that determine how spatial inhomogeneities in near-equilibrium macroscopic systems relax via hydrodynamic transport. The resulting predictions, known as Green-Kubo relations, equate these macroscopic transport coefficients to the microscopic equilibrium correlation functions of local current observables. This is central to our theoretical description of weakly nonequilibrium systems and underlies a number of experimental techniques for probing materials properties, such as microrheology and light scattering. Active systems, however, are nonequilibrium, and far from equilibrium the FDT becomes an inequality. 
One of the interesting directions is identifying classes of perturbations whose response verifies an equilibriumlike fluctuation-response equality. This, in principle, will allow us to extract linearized hydrodynamic transport equations around homogenous nonequilibrium steady states [160,161].

A signature of every nonequilibrium system is current, for instance, heat flux down a temperature gradient. Recently, a new kind of nonequilibrium principle - a thermodynamic uncertainty relation - has been proposed that demonstrates how energy dissipation continues to constrain current fluctuations far from equilibrium $[162,163]$. This novel principle relates the heat dissipated and the variance of the current fluctuations, offering a remarkable bound on response coefficients out of equilibrium akin to the equilibrium fluctuation-dissipation theorem. The thermodynamic uncertainty relation is implemented to quantify work and dissipation cycles within emergent strain waves in chiral active matter [164]. Exploring its implications in other active context and formulating other general relations of this type are important open challenges.

Since all equilibrium systems satisfy the FDT, an observed departure in the absence of visible external forcing signals an underlying active process. This basic principle is utilized to reveal nonequilibrium activity [165]. Often, deviations from the FDT are characterized by introducing a frequency-dependent effective temperature through the relation [cf. Eq. (2)]

$$
T(\omega)=\frac{1}{k_{B}} \frac{2 \omega S(\omega)}{\chi^{\prime \prime}(\omega)} .
$$

One might suspect that this relation reveals frequencies (timescales) relevant to the nonequilibrium behavior $[166,167]$. There is, however, no general principle that allows us to use this quantity to investigate the underlying microscopic mechanism. Insight is usually gained in this context in conjunction with modeling $[165,168-170]$. Note that $T(\omega)$ can even be negative [171].

Thus, the development of new model-independent frameworks that can be used to identify the scales of energy dissipation is crucial for a mechanistic understanding of nonequilibrium processes in active-matter systems.

\section{Thermodynamic arrow of time}

Nonequilibrium thermodynamics makes precise quantitative predictions about how time-reversal asymmetry and energy utilization (or dissipation) are manifested in nonequilibrium fluctuations [159]. Some of this theory's most prominent results are the fluctuation theorems, a collection of symmetries of the fluctuations of thermodynamic quantities such as the heat flow between a system and its environment. The fluctuation theorems have proven to be a very powerful tool to gain information from small systems where traditional thermodynamics does not apply [172]. Whether these frameworks can be applied to multiscale, complex systems such as active matter to provide novel insight is yet unknown and an exciting direction [130].

A cornerstone of stochastic thermodynamics is a quantitative connection between physical energy dissipation and the statistical irreversibility (arrow of time) of the dynamics.

To be specific, imagine we make a sequence of measurements of a physical observable every $\tau$ seconds and collect them into a list, or trajectory, $\gamma_{\tau}=\left(x_{\tau}, x_{2 \tau}, \ldots x_{n \tau}\right)$. These could be anything from the position of a particle to densities. Thermal fluctuations make the measurements noisy, and in each realization of this experiment we obtain a different sequence of outcomes, which we characterize with a probability distribution $\mathcal{P}\left(\gamma_{\tau}\right)$. In fact, it is possible, if not uncommon, to observe a previous sequence in the exact reverse order, $\tilde{\gamma}_{\tau}=\left(x_{n \tau}, \ldots, x_{2 \tau}, x_{\tau}\right)$, which occurs with probability $\mathcal{P}\left(\tilde{\gamma}_{\tau}\right)$. Stochastic thermodynamics teaches us that these fluctuations actually are not just incoherent noise but, in fact, constrain the physical heat dissipation (or energy consumption) $\dot{Q}_{\text {diss }}$ of the nonequilibrium system. Formally, we compare the probabilities of observing any sequence of measurements and its reverse through a information-theoretic metric of distinguishability $[173,174]$, which is called the relative entropy rate, $\dot{D}_{\tau}=\lim _{n \rightarrow \infty}(1 / n) \int \mathcal{P}\left(\gamma_{\tau}\right) \ln \left[\mathcal{P}\left(\gamma_{\tau}\right) / \mathcal{P}\left(\tilde{\gamma}_{\tau}\right)\right] \mathcal{D}\left[\gamma_{\tau}\right]:$

$$
\dot{Q}_{\text {diss }} \geq k_{B} T \dot{D}_{\tau},
$$

where $k_{B}$ is Boltzmann's constant and $T$ is the temperature of the surroundings. As the relative entropy $\dot{D}_{\tau}$ measures how distinguishable the processes is from its reverse, we call it the irreversibility. In other words, it quantifies the direction of the arrow of time. Equation (4) reveals a fundamental relationship between how irreversible a processes is-obtained from passive measurements of the dynamics - to the rate of energy consumption. This applies to any observable and nearly any nonequilibrium steady state. The irreversibility metric has proven to be a robust experimental and computational tool to detect nonequilibrium activity even in the absence of observable flows [173-179].

It is important to note that the connection between irreversibility and dissipation in active matter [132] is not as straightforward as in passive matter [159], and it is unclear whether we can apply this powerful framework to complex many-body active-matter systems. Interestingly, this leads to a plethora of definitions for irreversibility, each providing distinct information about the nonequilibrium nature of the dynamics. Current efforts are twofold: (i) the analytical and numerical studies of irreversibility and dissipation in theoretical models, where some exact analytical expressions can be derived, either in field theories $[154,167]$ or in particle-based models [180], and (ii) other studies which develop indirect methods for estimating irreversibility and dissipation, without any a priori 
knowledge of the underlying equations of motion ruling the dynamics. This is a very exciting direction, and very recently it has been demonstrated experimentally that one can extract quite a bit more information from this fundamental principle by using a new method, namely, multiscale statistical irreversibility, which can yield the scales of energy consumption in active systems. It is observed that the measured irreversibility changes with measurement frequency [181], in a manner correlated with a characteristic timescale of the underlying energy-consuming process. Thus, by analyzing statistical irreversibility over different time and length scales, we can discern the characteristic features of how energy is used in active matter without building a model. Other efforts are local measures of irreversibility [182] as well as exploring machine-learning approaches [183].

And this is just the beginning. Correlating such dataanalysis techniques with observations of function and structure can offer a principled method to characterize energy dynamics in complex matter. This also raises the question whether there is a scale at which dissipation is maximum and whether it is possible to use effective equilibrium descriptions beyond these scales $[180,184]$.

Looking forward, it will be exciting to see how these frameworks can provide a quantitative understanding of how thermodynamics dictates structure in active matter and function in living systems.

\section{NONRECIPROCITY AND CHIRALITY}

One of the most rapidly growing areas of active-matter physics is the study of situations where the nonequilibrium nature of the system results in the apparent violation of fundamental physical laws. Two important examples are nonreciprocity and chirality. Nonreciprocal forces that seemingly violate the equality of action and reaction arise when interactions are mediated by a nonequilibrium medium and can lead to new time-dependent self-organized states $[123,124,185,186]$. Active forces can also break the symmetry of the response of a system to external perturbation, resulting, for instance, in so-called odd elasticity [187]. Nonreciprocity and chirality are intimately related and pervasive in active matter.

\section{A. Nonreciprocal interactions drive new emergent behavior}

Newton's third law establishes that pair interactions among parts of a mechanically isolated system are reciprocal: For every action, there is an equal and opposite reaction. Such reciprocity applies to all systems where interactions can be derived from a Hamiltonian and governs all microscopic physical interactions. Reciprocity of interactions can also be seen as a consequence of detailed balance in a Markovian dynamics governed by a master equation. Just requiring a time-reversal-invariant steady state guarantees that the dynamics is downhill in the space described by a function of configurations that can be interpreted as energy. A natural definition of forces as gradients of this energy follows, ensuring reciprocity. In physical systems, nonreciprocity often emerges when effective interactions among mesoscopic parts of a system are mediated by a nonequilibrium medium, as in plasmas $[188,189]$ and mixtures of diffusiophoretic colloids [190,191]. Nonreciprocal interactions are ubiquitous in active and living systems that break detailed balance at the microscale, from social forces $[192,193]$ to promoterinhibitor couplings among cell types in developing organs and organisms [194], to antagonistic interspecies interactions in bacteria [195] and prey-predator systems [196]. Understanding how nonreciprocity (NR) affects nonequilibrium phase transitions and emergent states of active matter is a rapidly growing research focus in the field.

\section{How do we define nonreciprocity?}

This important question is usefully answered in a restricted context in which a notion of force operates and the evasionnot violation, of course - of Newton's Third Law can be appreciated. To fix ideas, let us work with dynamical variables $\left\{x_{a}, a=1,2, \ldots, N\right\}$, even under time reversal. These could be the positions or orientations of $N$ particles labeled by $a$. Let us assume they undergo inertialess dynamics governed purely by force balance:

$$
\Gamma_{a b} \frac{d x_{b}}{d t}=\sum_{b} F_{a b}+f_{a}
$$

in a dissipative medium with a matrix of kinetic coefficients with components $\Gamma_{a b}$, velocity-independent interaction forces $F_{a b}$, and Gaussian white noise $f_{a}$ with $\left\langle f_{a}(0) f_{b}(t)\right\rangle=$ $2 D_{a b} \delta(t)$. If this were a thermal equilibrium problem, we would have $D_{a b} \propto \Gamma_{a b}$. Nonreciprocality can enter if $\Gamma_{a b} \neq \Gamma_{b a}$, or $F_{a b} \neq-F_{b a}$, or both, violating the symmetry properties of kinetic coefficients [197] and/or static inverse susceptibilities. This is not a fundamental violation of Newton's Third Law, as the system is in contact with a damping medium. However, if the system were governed by an energy function that depended only on the relative values of the $\left\{x_{a}\right\}, F_{a b}+F_{b a}$ would necessarily be zero despite the presence of a medium that could take up the slack. Outside a context where the dynamics can be formulated in terms of forces or torques, a more general notion of nonreciprocity is still useful, in the form of an absence of $a \leftrightarrow b$ symmetry in the sensitivities of $\dot{x}_{a}$ to changes in $x_{b}: d x_{a} / d t=C_{a b} x_{b}+\cdots$ with $C_{a b} \neq C_{b a}$ [186]. However, the relation to Onsager reciprocity, and the equilibrium limit itself, is precise only when the dynamics is formulated in the detailed form of Eq. (5). We turn now to specific realizations of nonreciprocity that are considered in the active matter context. 


\section{Nonreciprocity and symmetry of the order parameter}

The simplest description of phase separation of a binary mixture is through the classical Cahn-Hilliard equations that describe the interdiffusive dynamics of two coupled conserved scalar concentration fields, $\phi_{a}$, for $a=A, B$, in one dimension [123]. The evolution of each concentration is governed by a $\phi^{4}$ field theory that allows for a spinodal instability according to Model $B$ dynamics:

$$
\partial_{t} \phi_{a}=\partial_{x}\left(\partial_{x} \frac{\delta F_{a}}{\delta \phi_{a}}+\kappa_{a b} \partial_{x} \phi_{b}\right),
$$

with

$$
F_{a}=\frac{1}{2} \int_{\mathbf{r}}\left(\chi_{a} \phi_{a}^{2}+\frac{1}{6} \phi_{a}^{4}+\gamma_{a}\left(\partial_{x} \phi_{a}\right)^{2}\right)
$$

When $\kappa_{a b}=\kappa_{b a}$, such a system undergoes bulk phase separation from a homogeneous mixed phase into two coexisting phases, each rich in one of the species. Recent work examines nonreciprocality arising from nonsymmetric, and, therefore, nonequilibrium, off-diagonal contributions to the effective chemical potential that cannot be derived from a free energy, leading to $\kappa_{A B} \neq \kappa_{B A}[123,124,185]$. Such a situation can arise, for instance, in mixtures of active colloids from the breaking of detailed balance in the microdynamics that is a defining feature of active systems. It is shown that sufficiently strong nonreciprocity can set the phase-separated state into motion, resulting in traveling density waves that break orientational symmetry. This work demonstrates that NR provides a generic mechanism for traveling, and possibly stable oscillatory patterns, where the two components play a chase-and-run game with each other, eventually settling into a stable spatiotemporally modulated structure. NR can additionally arrest the phase separation, turning the spinodal decomposition into a Turing-type instability with length scale selection [185]. While the emergence of spatial and temporal patterns is well known, for instance, in models of population dynamics with antagonistic or mutualistic reproduction rates [198,199], it is surprising in systems described by conserved scalar fields with purely diffusive dynamics.

Related work examines the effect of nonreciprocity in a two-species Vicsek model characterized by coupled vector order parameters that encode the mean velocity of each species [186]. Nonreciprocity is introduced here by assuming that, while species $A$ aligns with both $A$ and $B$, species $B$ aligns with $B$ but antialigns with $A$ (see Fig. 6). One then finds a dynamical chiral state, with no equilibrium counterpart where the mean velocities of each species rotate either clockwise or counterclockwise, maintaining a constant phase difference- a vectorial analog to the traveling states of phase separated scalar Cahn-Hilliard fields $[123,185]$.

In both examples discussed above, NR introduces new time-dependent collective states that dynamically restore broken symmetries of the reciprocal system. NR sets patterns
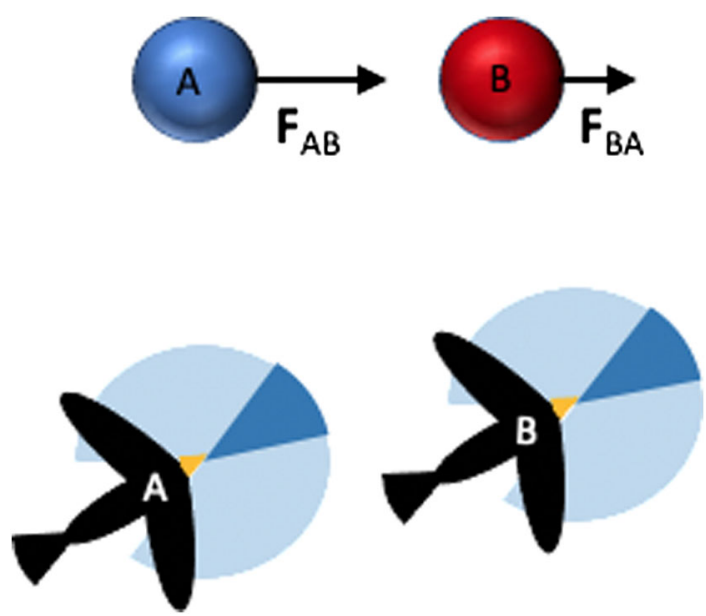

FIG. 6. Examples of nonreciprocal interactions. Top: Active colloids can experience NR effective interactions due to different surface reactivities. Here, particle $A$ is attracted to $B$, but $B$ is repelled by $A$. Bottom: Birds interact with other birds that are within their vision cone. Here, bird $A$ interacts with $B$, which is within the vision cone of $A$, but $B$ does not interact with $A$.

of scalar fields into motion, effectively breaking polar symmetry, and endows antagonistically coupled polar flocks with handedness. More generally, what are the consequences of NR in pattern-forming systems with scalar, polar, or nematic symmetry? How does NR affect the emergent behavior and the nature of the phase transitions between states? Also to be explored is the role of boundary conditions that may play a special role in systems with nonreciprocal interactions. Finally, the drift instability of scalar model described above as well as the transition of the two-species Vicsek model appear to be examples of a general class of $P T$ breaking $(P=$ parity, $T=$ time reversal) transitions that appear in open quantum systems with non-Hermitian Hamiltonian operators, resulting in time-periodic Floquet states [200-202]. Exploring the connection between nonreciprocal active matter and non-Hermitian quantum mechanics is an important emerging direction.

\section{Nonreciprocity in active solids}

The role of nonreciprocity in mesoscale effective interactions is also highlighted in the context of the elasticity of materials where the interactions among the individual constituents are nonconservative [187]. In this case, nonreciprocity manifests itself in the form of antisymmetric (odd) elastic moduli that are needed to characterize the linear elasticity of isotropic solids, in addition to familiar shear and compressional moduli. The presence of these odd response functions yields new nonequilibrium behavior, such as the ability of an inertialess elastic solid to support elastic wave propagation, as well as auxetic responsewhen stretched, the material expands in the direction perpendicular to the applied force. Note that this last can 
arise in equilibrium systems as well, through a negative Poisson ratio.

Even in the absence of chirality, and, hence, of odd elasticity, the effective pair interaction of orientable motile particles in an elastic medium is NR, because it is mediated by the reorienting effect of the strain field that their motion generates. The result is a strongly nonmutual "tactic" [203] interaction between two particles of the same type, distinguished only by which one lies ahead as defined by the direction in which it points. The particle at the rear acquires a purely mechanical "stealth" and can sense and move toward the one in front without signalling its presence [204].

\section{Nonreciprocity, activity, and information}

It seems clear that nonreciprocity is generic in active matter, but whether activity and nonreciprocity share the same fundamental origin is yet to be understood. Consider two coordinates $x_{1}$ and $x_{2}$ in a system rendered active by maintaining a constant positive chemical potential difference $\Delta \mu$ between the reactants and products of a chemical reaction. Within the standard active-matter paradigm $[12,167,205,206]$, active dynamics arises through chemomechanical cross-couplings $\zeta_{i}, i=1,2: \dot{x}_{i}=\zeta_{i} \Delta \mu+\cdots$, where the ellipsis denotes the passive part of the dynamics and, in general, the $\zeta_{i}$ depend on $x_{1}$ and $x_{2}$. Unless forbidden by additional symmetries, in general, $\partial \zeta_{i} / \partial x_{j} \neq \partial \zeta_{j} / \partial x_{i}$, so the dynamics of $x_{1}$ and $x_{2}$ should be nonreciprocal. Agent-based models like the iconic Vicsek model may be based on reciprocal interactions or alignment rules but upon coarse-graining are described by continuum equations with macroscopic couplings that do not respect Onsager's relations and, hence, break macroscopic nonreciprocity. Indeed, nonreciprocity rather than motility can be seen [207] to lie at the heart of the Vicsek and the Toner-Tu models. If the orientation vector carried by a particle aligns more strongly with that of its neighbor ahead of it than with its neighbor behind it, where ahead and behind are defined with respect to the direction of the focal particle's orientation vector, the polar order parameter in the coarse-grained theory advects itself as if it were a velocity even if the particles are not motile [207]. The directed information transfer associated with this advective nonlinearity assures long-range order in two dimensions. It was also recently shown that the interpretation of active hydrodynamics as a continuum theory with couplings that do not satisfy Onsager's relations can provide useful insights on energy dissipation and irreversibility [208]. Demonstrating in detail how such macroscopic nonreciprocity arises from microscopic dynamics with broken TRS but without explicit nonreciprocity remains an open challenge. For instance, coarse-graining often involves empirical closures and is generally carried out by neglecting noise, which is then added back phenomenologically in the continuum model, presumably unconstrained by a relation to kinetic coefficients. Could this type of breaking of the fluctuation-dissipation relation lead generically to the emergence of nonreciprocity in the continuum dynamics?

Finally, NR may be the key to understanding directed information transmission in living systems, such as signalling in cell biology or communication in social environments. In synthetic active matter and robotics, NR interactions can be used for control of time-delayed feedback, memory, and information flow. The understanding of these processes is an emerging direction with far reaching implications from biology to engineering.

\section{B. Chiral active matter}

An object is conventionally termed chiral [209-212] if it cannot be superimposed on its image in a plane mirror by means of rigid motions. Chirality can enter through structure, as in a helix in three dimensions or a scalene triangle in two, or dynamically, as in a spinning object such as a rotary molecular motor. In $2 \mathrm{D}$, chirality is uniquely defined with respect to an axis normal to the plane in which the system lives, such as the clockwise (CW) or counterclockwise $(\mathrm{CCW})$ sense of rotation of a spinner. In 3D, however, the handedness of an object depends, quite literally, on one's point of view [213]. Indeed, an object that is three-dimensionally achiral in an absolute sense can nonetheless display chiral behavior in its dynamical response about a given axis [214]. Chirality is inescapable in biology, and living matter is active, so it is natural to explore the interplay of chirality and activity.

\section{Chirality is an asymmetry}

Chirality describes the absence of a spatial symmetry. Asymmetry, in active systems, begets spontaneous motion and governs its direction. A pragmatic approach to chiral active systems thus emerges, through time-reversalbreaking stresses and currents constructed from local fields and their gradients, with an odd number of appearances of the Levi-Civita tensor. Chirality pertains to spatial, and activity to temporal, asymmetry. One reason to study the two together is that in passive systems chirality tends not to reveal its presence in long-wavelength mechanical properties-for example, the elastic and hydrodynamic properties of cholesteric liquid crystals at equilibrium map exactly to those of smectics [215,216]. In achiral systems with translational order, active forces introduce terms whose form superficially resembles those already present in the corresponding passive systems [217] or created by static external fields [218,219]. Taken together, however, the effects of chirality and activity can reinforce each other, with surprising consequences. Some of these can be seen in the sampling of results below, but we expect much richness from this interplay in future studies of chiral active matter. 


\section{Turners and spinners: Dry chiral active matter}

We begin with agent-based models without an ambient fluid. Motile particles whose heading turns at a constant rate $[220,221]$ provide a simple realization of chiral, polar active matter, displaying enhanced order and, for rapid enough turning rate, microphase separation into coherently rotating domains with the scale set by the turning radius. Persistently spinning particles allow the study of activity without translational motility or even an axis of alignment. The particles could be permanent spinners [222] or actuated by a rotating field [223], in which case chirality is a consequence of rotation, or they could convert incoherent energy input into rotary motion by virtue of their chiral shape [224]. These active but nonmotile spinners give rise to a rich range of phenomena. Spinners in a dense passive monolayer display a long-ranged interaction whose character changes as the layer changes from fluid to solid [223], while two-dimensional crystalline phases of spinners display one-way propagating edge currents [222].

A potentially important question of principle in these dry chiral active systems concerns how much, and under what circumstances, the circle-walker system, which has a local polar order parameter but no long-range vector order, differs from the spinner system. How do properties vary upon tuning the turning direction from clockwise to counterclockwise as one crosses the point of infinite turning radius where a uniform flock intervenes? In a more speculative vein, can elementary active-matter models with chirality offer novel approaches to exploring chiral discrimination and proofreading-life-or-death issues during protein synthesis in the cell [225]? In this connection, recent experiments [226] on a mixture of left- and rightturning motile ellipsoids are noteworthy for placing in evidence an active mechanism for stereoselection, through the formation of a preponderance of motile achiral dimers and a smaller fraction of spinning chiral dimers.

\section{Living chiral fluids}

The description of wet chiral active systems (see footnote 3) originates in Ref. [227] for bulk and Ref. [228] for thin-film settings. Reference [227] considers suspensions of torque dipoles consisting of a pair of oppositely directed point torques perpendicular or parallel to their separation vector, as well as chiral force dipoles made of a pair of oppositely directed point forces perpendicular to their separation vector, compensated by a torque monopole to ensure zero total torque. The resulting extended hydrodynamic equations, including an angular momentum density, predict that the intrinsic rotation and the vorticity can differ even in steady state and that a confined active chiral fluid with polar order can produce macroscopic shear [229]. The spontaneous rotation of chiral active fluids yields an escape route [230] from the generic instability of Ref. [42].
Arguably the most far-reaching implications of wet chiral active hydrodynamics are for developmental biology, specifically on the emergence of macroscopic left-right asymmetry in the development of a multicellular organism. Experiments on the development of the nematode Caenorhabditis elegans, informed by the coarse-grained theory of a thin film of chiral active fluid, show that counterrotating flows arising from cytoskeletal stresses and torques at the scale of a cell lead to asymmetry at the scale of the embryo, in particular, cell lineages $[231,232]$. Major open directions include identifying the (macro)molecular players responsible for the operative torque generation and the mechanism that organizes these coherently to yield the macroscopic torques and flows.

A dramatic example of chiral organization in biology is provided by recent experiments on dense collections of starfish embryos at an interface [164]. Over the course of their natural development, thousands of swimming embryos come together to form living chiral crystal (LCC) structures that persist for many hours (see Fig. 7). The self-assembly, dynamics, and dissolution of these LCCs are controlled entirely by the embryo's internal developmental program. Starfish embryos are inherently chiral, as they spin about their animal pole axis in a handed manner. When self-
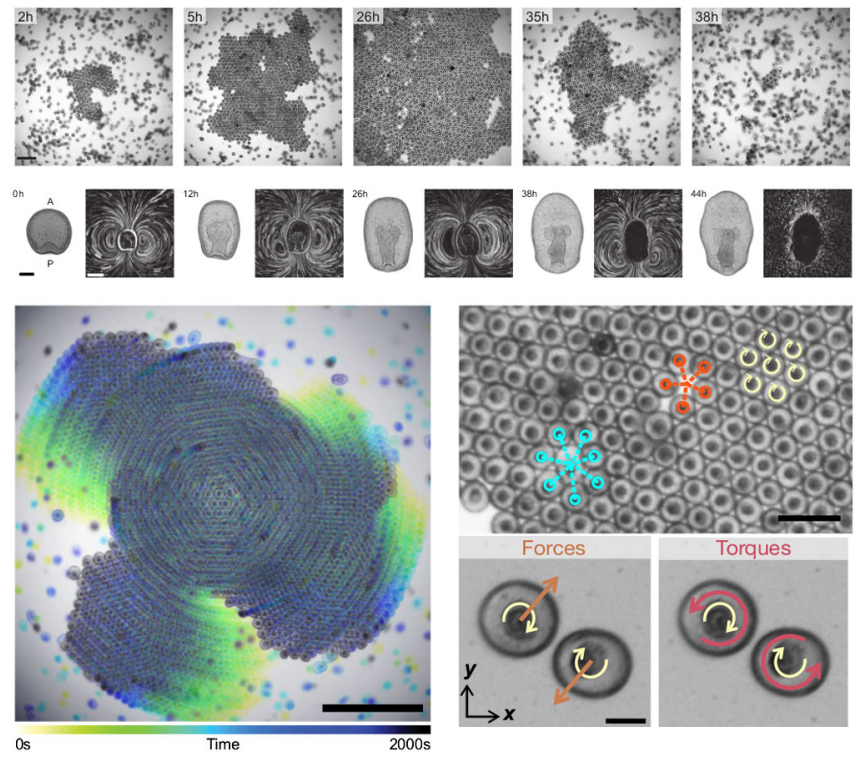

FIG. 7. Developing starfish embryos self-organize into living chiral crystals. Time sequence of still images showing crystal assembly and dissolution $(t=0 \mathrm{~h}$ corresponds to the onset of clustering; scale bar, $1 \mathrm{~mm}$ ). Embryo morphology and flow fields change with developmental time (shape scale bar, $100 \mu \mathrm{m}$; flow field scale bar, $200 \mu \mathrm{m}$ ). Embryos assembled in a crystal perform a global collective rotation (scale bar, $2 \mathrm{~mm}$ ). Spinning embryos (yellow arrows) in the crystal form a hexagonal lattice, containing fivefold (red) and sevenfold (cyan) defects (scale bar, $0.5 \mathrm{~mm}$ ). Spinning embryos exchange forces (brown arrows) and torques (red arrows) due to hydrodynamic interactions. Adapted from Ref. [164]. 
organized into a cluster, a fraction of each embryo's torque is transferred to the whole crystal, resulting in a global cluster rotation. Perhaps more importantly, the chiral spinning motions also lead to transverse interactions and torque exchanges between embryo pairs. Because of the nonreciprocal nature of these interactions, LCCs can support self-sustained chiral waves and shear cycles, similar to those recently predicted in odd elastic materials, providing evidence for the importance of nonreciprocality in multiorganismal living matter. Since many multicellular systems naturally break time-reversal and chiral symmetries in a manner similar to the starfish embryo system, this work can open up an exciting avenue in search of odd properties in biophysical systems.

\section{Translational order, bulk versus boundary and dimensionality}

Odd elasticity of active solids [187] is an especially dramatic manifestation of the combined effects of chirality and activity. A linear elastic tensor $C_{a b c d}$ relates stress to strain: $\sigma_{a b}=C_{a b c d} U_{c d}$, but the absence of an energy function liberates $C_{a b c d}$ from the constraint of symmetry under $a b \leftrightarrow c d$. Among the consequences are work extraction from the active solid in quasistatic cycles and propagating modes in the nominally inertia-free regime. Odd viscoelasticity is discussed in Ref. [233].

Active chiral systems with one-dimensional translational order-active cholesterics [219] — display a unique nonreciprocal effect: Gradients of layer curvature evoke a response in the perpendicular in-plane direction, like an odd Laplace pressure gradient. Whereas the odd elastic force density of two-dimensional chiral active solids reflects an antisymmetric contribution to the linear relation between stress and strain, the force density of Ref. [219] arises even when the strain is zero. If such a system goes through a Helfrich-Hurault [234,235] undulational instability or its active counterpart [218,236], this effect, odder than odd elasticity, produces a columnar array of fluid-flow vortices, with an "antiferromagnetic" spatial pattern of vorticity [219].

In these translationally ordered systems, the combined effects of chirality and activity manifest themselves in the bulk. By contrast, the most striking features of chiral active fluids seem to lie in their edge modes [222]. Understanding the fundamental reason for this contrast in behaviors and an exploration of possible connections to the expulsion of chirality to the edge in a layer of three-dimensionally chiral particles [237] are interesting open directions.

Lastly, studies of chiral active systems largely focus on two dimensions, where chiral effects can be viewed in the simple CW-CCW dichotomy which links naturally to persistent currents. Understanding how activity and chirality combine in three-dimensional systems, especially in the absence of a preferred direction with respect to which to project to two dimensions, is a challenge. For purely viscous 3D odd fluids, see Ref. [238]. It is clear that chirality provides unusual opportunities for the manifestation of active effects on large spatial scales and that explorations of the interplay of activity and chirality will be a major theme in the study of active systems for years to come.

\section{ACTIVE MATTER IN BIOLOGY}

Living cells are active entities capable of a number of self-driven mechanical functions, such as shape changes, motility, and division. Through interactions and coupling to the environment, they assemble into biological tissue, forming organs and organisms with entirely new emergent behavior. An important open question in active matter physics is developing a predictive continuum theory of living matter that relates subcellular and cell-scale processes to adaptive mechanics at the tissue scale. A key challenge is relating the coefficients of the sought-after continuum models to both the parameters of mesoscopic models, such as Vertex, Voronoi, or multiphase field models, and to quantities controlled in experiments. A measure of success of the theory will be its ability to identify classes of molecular signaling mechanisms that define specific effective material parameters capable of characterizing behaviors at the organ and organism scale.

\section{A. Coupling between multiple fields underlies the dynamics of tissues}

An approach to developing such a theory finds its inspiration in a successful paradigm in developmental biology. The essence of this paradigm is the notion that spatial and temporal concentration patterns of diffusable chemicals known as morphogens specify the organization of cells into emergent structures $[137,138,239]$. In recent years, the traditional notion of scalar concentration fields has acquired broader scope, as it has become evident that organization at the tissue scale can often be described in terms of the dynamics of continuum supracellular fields that often include orientational degrees of freedom and define the tissue's mechanical behavior [240-242]. An important example is Planar Cell Polarity that describes the tendency of epithelial cells to polarize due to anisotropic protein distribution within a given cell [243]. Cell-cell interactions can then coordinate such polarity at the tissue level, resulting in large-scale tissue anisotropy usefully described in terms of spatial and temporal variation of a continuum vector field. Such an approach provides the opportunity to take advantage of the machinery of the physics of flocking, and it acquires quantitative power when the continuum field can be related to specific cellular processes.

Another application of these ideas to living matter is motivated by the recent observation of nematic order in a 
variety of biological settings, as discussed in Sec. II C. In some cases, as in layers of spindle-shaped progenitor neural cells [34], the individual cells are clearly elongated and nematic textures can readily be associated with the arrangements of cellular shapes, suggesting that orientational order may be driven by crowding through the interplay of steric and entropic effects, much like in equilibrium. In other systems, such as the Drosophila embryo [241] or the freshwater organism Hydra [37], nematic order is evident in the organization of supracellular myosin or actin fibers. Less clear are the origin and signature of nematic order in epithelial layers, such as MDCK cells [35]. In general, more work is needed to understand what may be the mechanical or biochemical processes that drive and control the formation of nematic textures and the biological role of such orientational order.

\section{B. Forces shaping tissues: From intracellular to cellular}

A related question concerns the nature of active forces in biological tissue. Individual cells crawl on substrates by generating contractile active stresses through the actomyosin machinery of their cytoskeleton. Recent experiments indicate, however, that, when such cells organize in confluent epithelia, interactions mediated by e-cadherins result in pulling forces exerted by cells on their neighbors, with extensile stresses at the tissue scale [244]. What are the relative roles of polar traction forces exerted by cells on a substrate or the surrounding medium and cell-cell "tractions" in controlling the extensile or contractile and polar or apolar nature of active stresses in tissue and the resulting modes of collective cell dynamics?

\section{Spatiotemporally varying material properties}

A key limitation of the continuum modeling approach lies in the assumption of fixed material properties of tissues, which is encoded in the choice of a particular constitutive law. Tissues are able to adapt their mechanical response to perturbations (both external and internal) and are characterized by multiple relaxation times. This demands a rheological model capable of capturing both active solidlike and fluidlike behavior in different regimes of stress response and to dynamically transition between the two. In other words, any rheological model of tissue mechanics must incorporate the active feedbacks between cellular mechanics, polarized motility, and the regulatory biochemistry of actomyosin contractility. These couplings play an essential role in the transmission of spatial information in large cell monolayers, which are often mediated by traveling waves, pulses, and a tug of war between cellcell and cell-substrate forces [245,246]. While some recent progress has been made on incorporating these couplings in continuum models [247], informed by studies of mesoscopic models and by experiments, formulating an adaptive rheological model of tissue remains an open challenge. This is further complicated by the fact that living tissue is also capable of adapting its mechanical state in response to changes in environment, through feedback loops in a way that has so far largely eluded predictive theoretical descriptions.

\section{Form meets function}

There are also situations where epithelial cells organize in remarkable orderly patterns that seem to be essential to the functioning of many tissues. Examples are photoreceptor cells in the eyes [248], the hexagonal cell packings in the wing of developing Drosophila [249] (see Fig. 8), and the remarkable rectangular cell lattice observed in the development of the freshwater shrimp Parhyale [250]. The mechanisms that control the development of such regular, ordered epithelial cell packings remain only partially understood. Do protein anisotropies existing at the single-cell or subcellular level control the emergence of ordered structures, as, for instance, suggested in models of the eye retina [251], or do regular cellular arrangements emerge spontaneously from cell-cell interactions? What is the role of growth and growth anisotropy in organizing ordered cell packings? Answering these questions may also help inform and guide new pathways of active assembly for the design of functional materials.

\section{E. Beyond broken symmetries}

Finally, in condensed matter physics, the notions of broken symmetry and conservation laws provide powerful principles for the identification of coarse-grained fields that allow the formulation of predictive continuum descriptions of complex phenomena. So far, the active matter community has largely been using the same ideas to formulate continuum models of active and living systems [240]. But living matter develops, divides, repairs itself, adapts to its environment, and evolves to perform specific functions. Its hierarchically organized constituents often compete for fixed pools of resources: For instance, in wound healing,

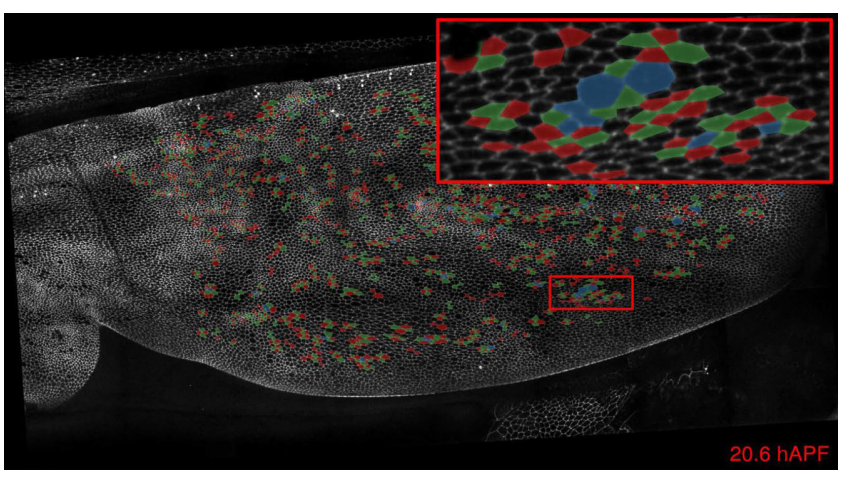

FIG. 8. Morphogenesis of the Drosophila pupal wing. The cells in the tissue undergo shape changes, cell divisions, cell rearrangements, and cell extrusions during wing morphogenesis. Colors denote cell contact dynamics during tissue morphogenesis. Adapted from Ref. [252]. 
the same actin pool may drive protrusive cell motility and cell contraction [253]. Can we identify general principles that may guide us in constructing field theories for this more complex type of matter, where spatial and temporal responses are often coupled and feed back onto each other? How do we build into our coarse-grained theories the fact that we are dealing with systems that have emerged from an evolutionary process, that they correspond to evolutionarily stable strategies? For example, when constructing a generic model of a physical system, one would never insist that parameter values should be poised at a threshold separating two qualitatively distinct behaviors. Such phase-transition points correspond to unstable fixed points of the renormalization group, so that this parameter choice would amount to nongeneric fine-tuning. But evidence from diverse living systems [254-257] suggests that tuning of this type may be an emergent result of evolution. And what is the influence of noise on the regulatory feedbacks that control cellular organization?

\section{THE FUTURE IS ACTIVE}

The field of active matter continues to evolve rapidly and to establish connections and relevance to many areas of science. In this article, we present a biased selection of topics where we expect significant progress to be made in the coming years. There are many other important emerging directions that are omitted. An example is the study of the interplay of motility and information transmission with models of active agents that change their state upon interaction with each other [258-260], as relevant, for instance, to epidemics spreading. Another is systems in which the active forcing can be viewed as maintaining temperature difference between two species of particles $[261,262]$.

The field of active matter started out by marrying the fluid dynamics of swimmers with the field theory of pattern formation and phase transitions. It has now acquired its own identity as a powerful framework for the description of spontaneous organization in both the living and the engineered worlds on a vast range of scales. The role of topology has become evident in active systems and has pointed to the possibility of deep connections with the statistical physics of open quantum systems [186,263].

It has also become evident that the topics discussed here are all interconnected, as displayed in Fig. 9, with many of these connections still awaiting quantitative exploration. The field of active matter was born from the physicist's ambition to use statistical physics and hydrodynamics to describe collective motion in the living world. The activematter framework has now had important successes in capturing examples of organization in living matter on scales from subnuclear to oceanic. The overall dream is to develop a predictive theory that allows us to design the hierarchical organization of active agents or "machines" into larger-scale machines tuned to perform specific

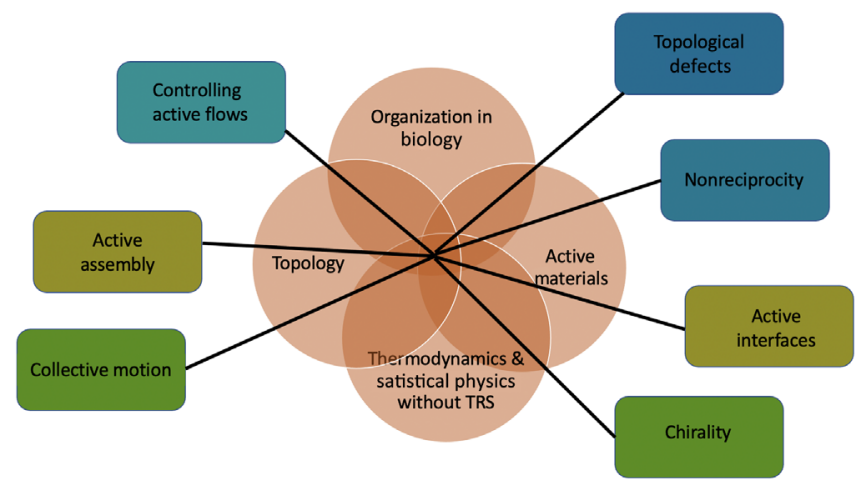

FIG. 9. A pictorial depiction of the connections among the various aspects of active-matter physics discussed in this article.

functions and to adapt to the task at hand. While nature does this every day, we are still far from achieving this goal but continue to make consistent progress.

\section{ACKNOWLEDGMENTS}

This article describes some of the outcomes and new directions of inquiry that emerged during the program Symmetry, Thermodynamics and Topology in Active Matter (Active20) at the Kavli Institute for Theoretical Physics (KITP) of UC Santa Barbara. The program, originally due to start on March 16, was at first halted by the Covid-19 pandemic. After the state of California issued a mandatory stay-at-home order on March 19, the KITP and the program coordinators quickly regrouped and on March 30 they started the first virtual KITP program which ran for nine weeks, with exceptionally high participation of scientists from all over the world, in spite of the challenges of time zones. This article presents the four authors' personal view, informed by contributions from all program participants, of emerging directions and open questions in active matter research. The authors thank all participants in Active 20 for their contribution to this article through presentations and discussions. The KITP program was supported by the National Science Foundation through Grant No. PHY1748958. The authors received additional support from the National Science Foundation under Grant No. DMR2041459 (M. C. M.) and through the Materials Science and Engineering Center at UC Santa Barbara, DMR-1720256 (iSuperSeed) (M. C. M. and M. J. B.). N. F. acknowledges National Science Foundation CAREER Grant No. PHYS1848247. S. R. acknowledges support from a J. C. Bose Fellowship of the SERB, India, and from the Tata Education and Development Trust.

[1] T. Vicsek, A. Czirók, E. Ben-Jacob, I. Cohen, and O. Shochet, Novel Type of Phase Transition in a System of Self-Driven Particles, Phys. Rev. Lett. 75, 1226 (1995). 
[2] J. Toner and Y. Tu, Long-Range Order in a TwoDimensional Dynamical XY Model: How Birds Fly Together, Phys. Rev. Lett. 75, 4326 (1995).

[3] C.W. Reynolds, in Proceedings of the 14th Annual Conference on Computer Graphics and Interactive Techniques (ACM Press, 1987), pp. 25-34.

[4] I. Aoki, A Simulation Study on the Schooling Mechanism in Fish, Nippon Suisan Gakkaishi 48, 1081 (1982).

[5] B. L. Partridge, The Structure and Function of Fish Schools, Sci. Am. 246, 114 (1982).

[6] S. Ramaswamy and R. A. Simha, The Mechanics of Active Matter: Broken-Symmetry Hydrodynamics of Motile Particles and Granular Layers, Solid State Commun. 139, 617 (2006).

[7] J. Prost and R. Bruinsma, Shape Fluctuations of Active Membranes, Europhys. Lett. 33, 321 (1996).

[8] S. Ramaswamy, J. Toner, and J. Prost, Nonequilibrium Fluctuations, Traveling Waves, and Instabilities in Active Membranes, Phys. Rev. Lett. 84, 3494 (2000).

[9] J. Darnell, H. Lodish, and D. Baltimore, Molecular Cell Biology, 2nd ed. (W.H. Freeman, 1990).

[10] B. A. Finlayson and L. E. Scriven, Convective Instability by Active Stress, Proc. R. Soc. A 310, 183 (1969).

[11] S. Ramaswamy, The Mechanics and Statistics of Active Matter, Annu. Rev. Condens. Matter Phys. 1, 323 (2010).

[12] M. C. Marchetti, J.-F. Joanny, S. Ramaswamy, T. B. Liverpool, J. Prost, M. Rao, and R. A. Simha, Hydrodynamics of Soft Active Matter, Rev. Mod. Phys. 85, 1143 (2013).

[13] T. Vicsek and A. Zafeiris, Collective Motion, Phys. Rep. 517, 71 (2012).

[14] M. Poujade, E. Grasland-Mongrain, A. Hertzog, J. Jouanneau, P. Chavrier, B. Ladoux, A. Buguin, and P. Silberzan, Collective Migration of an Epithelial Monolayer in Response to a Model Wound, Proc. Natl. Acad. Sci. U.S.A. 104, 15988 (2007).

[15] M. Ballerini, N. Cabibbo, R. Candelier, A. Cavagna, E. Cisbani, I. Giardina, V. Lecomte, A. Orlandi, G. Parisi, A. Procaccini, M. Viale, and V. Zdravkovic, Interaction Ruling Animal Collective Behavior Depends on Topological Rather Than Metric Distance: Evidence from a Field Study, Proc. Natl. Acad. Sci. U.S.A. 105, 1232 (2008).

[16] D. Helbing, I. Farkas, and T. Vicsek, Simulating Dynamical Features of Escape Panic, Nature (London) 407, 487 (2000).

[17] N. Bain and D. Bartolo, Dynamic Response and Hydrodynamics of Polarized Crowds, Science 363, 46 (2019).

[18] V. Narayan, S. Ramaswamy, and N. Menon, Long-Lived Giant Number Fluctuations in a Swarming Granular Nematic, Science 317, 105 (2007).

[19] J. Deseigne, O. Dauchot, and H. Chaté, Collective Motion of Vibrated Polar Disks, Phys. Rev. Lett. 105, 098001 (2010).

[20] N. Kumar, H. Soni, S. Ramaswamy, and A. K. Sood, Flocking at a Distance in Active Granular Matter, Nat. Commun. 5, 4688 (2014).

[21] Autophoresis denotes particle drifts induced by concentration gradients created by the particles themselves.

[22] R. Golestanian, Phoretic Active Matter, arXiv:1909.03747.

[23] C. Bechinger, R. Di Leonardo, H. Löwen, C. Reichhardt, G. Volpe, and G. Volpe, Active Particles in Complex and
Crowded Environments, Rev. Mod. Phys. 88, 045006 (2016).

[24] R. A. Simha and S. Ramaswamy, Hydrodynamic Fluctuations and Instabilities in Ordered Suspensions of SelfPropelled Particles, Phys. Rev. Lett. 89, 058101 (2002).

[25] R. Voituriez, J.-F. Joanny, and J. Prost, Spontaneous Flow Transition in Active Polar Gels, Europhys. Lett. 70, 404 (2005).

[26] H. H. Wensink, J. Dunkel, S. Heidenreich, K. Drescher, R. E. Goldstein, H. Löwen, and J.M. Yeomans, Meso-scale Turbulence in Living Fluids, Proc. Natl. Acad. Sci. U.S.A. 109, 14308 (2012).

[27] A. Bricard, J.-B. Caussin, N. Desreumaux, O. Dauchot, and D. Bartolo, Emergence of Macroscopic Directed Motion in Populations of Motile Colloids, Nature (London) 503, 95 (2013).

[28] P. M. Chaikin and T. C. Lubensky, Principles of Condensed Matter Physics (Cambridge University Press, Cambridge, England, 2000).

[29] T. Sanchez, D. T. N. Chen, S. J. DeCamp, M. Heymann, and Z. Dogic, Spontaneous Motion in Hierarchically Assembled Active Matter, Nature (London) 491, 431 (2012).

[30] Y.-H. Zhang, M. Deserno, and Z.-C. Tu, Dynamics of Active Nematic Defects on the Surface of a Sphere, Phys. Rev. E 102, 012607 (2020).

[31] D. Nishiguchi, K. H. Nagai, H. Chaté, and M. Sano, LongRange Nematic Order and Anomalous Fluctuations in Suspensions of Swimming Filamentous Bacteria, Phys. Rev. E 95, 020601(R) (2017).

[32] D. Dell'Arciprete, M. L. Blow, A. T. Brown, F. D. C. Farrell, Juho S. Lintuvuori, A. F. McVey, D. Marenduzzo, and W. C. K. Poon, A Growing Bacterial Colony in Two Dimensions as an Active Nematic, Nat. Commun. 9, 4190 (2018).

[33] Y. I. Yaman, E. Demir, R. Vetter, and A. Kocabas, Emergence of Active Nematics in Chaining Bacterial Biofilms, Nat. Commun. 10, 2285 (2019).

[34] K. Kawaguchi, R. Kageyama, and M. Sano, Topological Defects Control Collective Dynamics in Neural Progenitor Cell Cultures, Nature (London) 545, 327 (2017).

[35] T. . Saw, A. Doostmohammadi, V. Nier, L. Kocgozlu, S. Thampi, Y. Toyama, P. Marcq, C. T. Lim, J. M. Yeomans, and B. Ladoux, Topological Defects in Epithelia Govern Cell Death and Extrusion, Nature (London) 544, 212 (2017).

[36] C. Blanch-Mercader, V. Yashunsky, S. Garcia, G. Duclos, L. Giomi, and P. Silberzan, Turbulent Dynamics of Epithelial Cell Cultures, Phys. Rev. Lett. 120, 208101 (2018).

[37] Y. Maroudas-Sacks, L. Garion, L. Shani-Zerbib, A. Livshits, E. Braun, and K. Keren, Topological Defects in the Nematic Order of Actin Fibres as Organization Centres of Hydra Morphogenesis, Nat. Phys. 17, 251 (2021).

[38] A. Tiribocchi, R. Wittkowski, D. Marenduzzo, and M. E. Cates, Active Model H: Scalar Active Matter in a Momentum-Conserving Fluid, Phys. Rev. Lett. 115, 188302 (2015). 
[39] R. Singh and M. E. Cates, Hydrodynamically Interrupted Droplet Growth in Scalar Active Matter, Phys. Rev. Lett. 123, 148005 (2019).

[40] R. Alert, J. Casademunt, and J.-F. Joanny, Active Turbulence, arXiv:2104.02122.

[41] J. Toner, Y. Tu, and S. Ramaswamy, Hydrodynamics and Phases of Flocks, Ann. Phys. (Amsterdam) 318, 170 (2005).

[42] R. A. Simha and S. Ramaswamy, Hydrodynamic Fluctuations and Instabilities in Ordered Suspensions of SelfPropelled Particles, Phys. Rev. Lett. 89, 058101 (2002).

[43] A pictorial representation of this mechanism can be found, for instance, in Ref. [11].

[44] V. Steinberg, Elastic Turbulence: An Experimental View on Inertialess Random Flow, Annu. Rev. Fluid Mech. 53, 27 (2021).

[45] A. N. Kolmogorov, The Local Structure of Turbulence in Incompressible Viscous Fluid for Very Large Reynolds Numbers, Proc. R. Soc. A 434, 9 (1991).

[46] L. Giomi, Geometry and Topology of Turbulence in Active Nematics, Phys. Rev. X 5, 031003 (2015).

[47] K. R. Sreenivasan and R. A. Antonia, The Phenomenology of Small-Scale Turbulence, Annu. Rev. Fluid Mech. 29, 435 (1997).

[48] R. Chatterjee, N. Rana, R. A. Simha, P. Perlekar, and S. Ramaswamy, Inertia Drives a Flocking Phase Transition in Viscous Active Fluids, Phys. Rev. X 11, 031063 (2021).

[49] A. Doostmohammadi, M. F. Adamer, S. P. Thampi, and J. M. Yeomans, Stabilization of Active Matter by FlowVortex Lattices and Defect Ordering, Nat. Commun. 7, 10557 (2016).

[50] A. Doostmohammadi, J. Ignés-Mullol, J. M. Yeomans, and F. Sagués, Active Nematics, Nat. Commun. 9, 3246 (2018).

[51] G. Duclos, R. Adkins, D. Banerjee, M. S. E. Peterson, M. Varghese, I. Kolvin, A. Baskaran, R. A. Pelcovits, T. R. Powers, A. Baskaran et al., Topological Structure and Dynamics of Three-Dimensional Active Nematics, Science 367, 1120 (2020).

[52] J. Słomka and J. Dunkel, Spontaneous Mirror-Symmetry Breaking Induces Inverse Energy Cascade in 3D Active Fluids, Proc. Natl. Acad. Sci. U.S.A. 114, 2119 (2017).

[53] J. Słomka, P. Suwara, and J. Dunkel, The Nature of Triad Interactions in Active Turbulence, J. Fluid Mech. 841, 702 (2018).

[54] V. Soni, E. S. Bililign, S. Magkiriadou, S. Sacanna, D. Bartolo, M. J. Shelley, and W. T. M. Irvine, The Odd Free Surface Flows of a Colloidal Chiral Fluid, Nat. Phys. 15, 1188 (2019).

[55] Y. Shin and C. P. Brangwynne, Liquid Phase Condensation in Cell Physiology and Disease, Science 357, eaaf4382 (2017).

[56] C.-C. P. Caulfield, Open Questions in Turbulent Stratified Mixing: Do We Even Know What We Do Not Know?, Phys. Rev. Fluids 5, 110518 (2020).

[57] F. G. Woodhouse and R. E. Goldstein, Spontaneous Circulation of Confined Active Suspensions, Phys. Rev. Lett. 109, 168105 (2012).

[58] A. Kumar, A. Maitra, M. Sumit, S. Ramaswamy, and G. V. Shivashankar, Actomyosin Contractility Rotates the Cell Nucleus, Sci. Rep. 4, 3781 (2014).
[59] J. M. Kosterlitz and D. J. Thouless, Ordering, Metastability and Phase Transitions in Two-Dimensional Systems, J. Phys. C 6, 1181 (1973).

[60] D. L. Stein, Kosterlitz-Thouless Phase Transitions in TwoDimensional Liquid Crystals, Phys. Rev. B 18, 2397 (1978).

[61] L. Giomi, M. J. Bowick, P. Mishra, R. Sknepnek, and M. C. Marchetti, Defect Dynamics in Active Nematics, Phil. Trans. R. Soc. A 372, 20130365 (2014).

[62] L. Giomi, M. J. Bowick, X. Ma, and M. C. Marchetti, Defect Annihilation and Proliferation in Active Nematics, Phys. Rev. Lett. 110, 228101 (2013).

[63] L. M. Pismen, Dynamics of Defects in an Active Nematic Layer, Phys. Rev. E 88, 050502(R) (2013).

[64] S. Shankar, S. Ramaswamy, M. C. Marchetti, and M. J. Bowick, Defect Unbinding in Active Nematics, Phys. Rev. Lett. 121, 108002 (2018).

[65] F. Vafa, M. J. Bowick, M. C. Marchetti, and B. I. Shraiman, Multi-defect Dynamics in Active Nematics, arXiv: 2007.02947.

[66] S. Shankar and M. C. Marchetti, Hydrodynamics of Active Defects: From Order to Chaos to Defect Ordering, Phys. Rev. X 9, 041047 (2019).

[67] Dry active matter refers to particles moving in the absence of an ambient fluid, where dissipation is mainly controlled by frictional drag with an external medium. Conversely, "wet" active matter describes systems where dissipation is principally controlled by viscous stresses mediated by a suspending medium. The size of the viscous scale $\ell_{v}$ defined in Sec. II B relative to the system size controls the crossover between these two limits. When the medium is a fluid, the fluid-mediated interactions are referred to as hydrodynamic interactions.

[68] P. Srivastava, P. Mishra, and M. C. Marchetti, Negative Stiffness and Modulated States in Active Nematics, Soft Matter 12, 8214 (2016).

[69] E. Putzig, G. S. Redner, A. Baskaran, and A. Baskaran, Instabilities, Defects, and Defect Ordering in an Overdamped Active Nematic, Soft Matter 12, 3854 (2016).

[70] A. Patelli, I. Djafer-Cherif, I. S. Aranson, E. Bertin, and H. Chaté, Understanding Dense Active Nematics from Microscopic Models, Phys. Rev. Lett. 123, 258001 (2019).

[71] D. J. G. Pearce, J. Nambisan, P. W. Ellis, A. FernandezNieves, and L. Giomi, Orientational Correlations in Active and Passive Nematic Defects, Phys. Rev. Lett. 127, 197801 (2021).

[72] K. Thijssen, M. R. Nejad, and J. M. Yeomans, Role of Friction in Multidefect Ordering, Phys. Rev. Lett. 125, 218004 (2020).

[73] A. U. Oza and J. Dunkel, Antipolar Ordering of Topological Defects in Active Liquid Crystals, New J. Phys. 18, 093006 (2016).

[74] S. J. DeCamp, G. S. Redner, A. Baskaran, M. F. Hagan, and Z. Dogic, Orientational Order of Motile Defects in Active Nematics, Nat. Mater. 14, 1110 (2015).

[75] A. Doostmohammadi, S. P. Thampi, and J. M. Yeomans, Defect-Mediated Morphologies in Growing Cell Colonies, Phys. Rev. Lett. 117, 048102 (2016).

[76] A. G. DeVries, M. Brunnbauer, Y. Hu, M. A. Jackson, B. Long, T. B. Neltner, O. Uzun, H. B. Wunsch, and 
F. Stellacci, Divalent Metal Nanoparticles, Science 315, 358 (2007).

[77] R. Zandi, B. Dragnea, A. Travesset, and R. Podgornik, On Virus Growth and Form, Phys. Rep. 847, 1 (2020).

[78] G. Duclos, C. Erlenkämper, J.-F. Joanny, and P. Silberzan, Topological Defects in Confined Populations of SpindleShaped Cells, Nat. Phys. 13, 58 (2017).

[79] K. Copenhagen, R. Alert, N. S. Wingreen, and J. W. Shaevitz, Topological Defects Promote Layer Formation in Myxococcus Xanthus Colonies, Nat. Phys. 17, 211 (2021).

[80] T. Shimaya and K. A. Takeuchi, 3D-Induced Polar Order and Topological Defects in Growing Bacterial Populations, arXiv:2106.10954.

[81] T. Sarkar, V. Yashunsky, L. Brézin, C. B. Mercader, T. Aryaksama, M. Lacroix, T. Risler, J.-F. Joanny, and P. Silberzan, Crisscross Multilayering of Cell Sheets, bioRxiv 10.1101/2021.06.22.449403v1.

[82] L. A. Hoffmann, L. N. Carenza, J. Eckert, and L. Giomi, Defect-Mediated Morphogenesis, arXiv:2105.15200.

[83] T. H. Tan, J. Liu, P. W. Miller, M. Tekant, J. Dunkel, and N. Fakhri, Topological Turbulence in the Membrane of a Living Cell, Nat. Phys. 16, 657 (2020).

[84] J. Liu, J. F. Totz, P. W. Miller, A. D. Hastewell, Yu.-C. Chao, J. Dunkel, and N. Fakhri, Topological Braiding and Virtual Particles on the Cell Membrane, Proc. Natl. Acad. Sci. U.S.A. 118, e2104191118 (2021).

[85] H. Wioland, F. G. Woodhouse, J. Dunkel, J. O. Kessler, and R. E. Goldstein, Confinement Stabilizes a Bacterial Suspension into a Spiral Vortex, Phys. Rev. Lett. 110, 268102 (2013).

[86] G. Duclos, C. Blanch-Mercader, V. Yashunsky, G. Salbreux, J.-F. Joanny, J. Prost, and P. Silberzan, Spontaneous Shear Flow in Confined Cellular Nematics, Nat. Phys. 14, 728 (2018).

[87] F. C. Keber, E. Loiseau, T. Sanchez, S. J. DeCamp, L. Giomi, M. J. Bowick, M. C. Marchetti, Z. Dogic, and A. R. Bausch, Topology and Dynamics of Active Nematic Vesicles, Science 345, 1135 (2014).

[88] R. Sknepnek and S. Henkes, Active Swarms on a Sphere, Phys. Rev. E 91, 022306 (2015).

[89] S. Shankar, M. J. Bowick, and M. C. Marchetti, Topological Sound and Flocking on Curved Surfaces, Phys. Rev. X 7, 031039 (2017).

[90] P. W. Ellis, D. J. G. Pearce, Y.-W. Chang, G. Goldsztein, L. Giomi, and A. Fernandez-Nieves, Curvature-Induced Defect Unbinding and Dynamics in Active Nematic Toroids, Nat. Phys. 14, 85 (2018).

[91] S. Henkes, M. C. Marchetti, and R. Sknepnek, Dynamical Patterns in Nematic Active Matter on a Sphere, Phys. Rev. E 97, 042605 (2018).

[92] P. Guillamat, J. Ignés-Mullol, and F. Sagués, Control of Active Liquid Crystals with a Magnetic Field, Proc. Natl. Acad. Sci. U.S.A. 113, 5498 (2016).

[93] N. Kumar, R. Zhang, J. J. de Pablo, and M. L. Gardel, Tunable Structure and Dynamics of Active Liquid Crystals, Sci. Adv. 4, eaat7779 (2018).

[94] T. D. Ross, H. J. Lee, Z. Qu, R. A. Banks, R. Phillips, and M. Thomson, Controlling Organization and Forces in
Active Matter through Optically Defined Boundaries, Nature (London) 572, 224 (2019).

[95] X. Gong, A. Mathijssen, Z. Bryant, and M. Prakash, Engineering Reconfigurable Flow Patterns via SurfaceDriven Light-Controlled Active Matter, arXiv:2004.01368 .

[96] R. Zhang, S. A. Redford, P. V. Ruijgrok, N. Kumar, A. Mozaffari, S. Zemsky, A. R. Dinner, V. Vitelli, Z. Bryant, M. L. Gardel et al., Spatiotemporal Control of Liquid Crystal Structure and Dynamics through Activity Patterning, Nat. Mater. 20, 875 (2021).

[97] D. Saintillan, Rheology of Active Fluids, Annu. Rev. Fluid Mech. 50, 563 (2018).

[98] L. C. Emmanuel, J. M. Yeomans, and A. Doostmohammadi, Active Matter in a Viscoelastic Environment, Phys. Rev. Fluids 5, 023102 (2020).

[99] Y. Bozorgi and P. T. Underhill, Effects of Elasticity on the Nonlinear Collective Dynamics of Self-Propelled Particles, J. Non-Newtonian Fluid Mech. 214, 69 (2014).

[100] E. J. Hemingway, A. Maitra, S. Banerjee, M. C. Marchetti, S. Ramaswamy, S. M. Fielding, and M. E. Cates, Active Viscoelastic Matter: From Bacterial Drag Reduction to Turbulent Solids, Phys. Rev. Lett. 114, 098302 (2015).

[101] E. J. Hemingway, M. E. Cates, and S. M. Fielding, Viscoelastic and Elastomeric Active Matter: Linear Instability and Nonlinear Dynamics, Phys. Rev. E 93, 032702 (2016).

[102] G. Li and A. M. Ardekani, Collective Motion of Microorganisms in a Viscoelastic Fluid, Phys. Rev. Lett. 117, 118001 (2016).

[103] S. Liu, S. Shankar, M. C. Marchetti, and Y. Wu, Viscoelastic Control of Spatiotemporal Order in Bacterial Active Matter, Nature (London) 590, 80 (2021).

[104] M. Kramar and K. Alim, Encoding Memory in Tube Diameter Hierarchy of Living Flow Network, Proc. Natl. Acad. Sci. U.S.A. 118, e2007815118 (2021).

[105] M. Prakash and N. Gershenfeld, Microfluidic Bubble Logic, Science 315, 832 (2007).

[106] F. G. Woodhouse and J. Dunkel, Active Matter Logic for Autonomous Microfluidics, Nat. Commun. 8, 15169 (2017).

[107] N. Romeo, A. Hastewell, A. Mietke, and J. Dunkel, Learning Developmental Mode Dynamics from SingleCell Trajectories, arXiv:2103.08130.

[108] D. B. Brückner, P. Ronceray, and C. P. Broedersz, Inferring the Dynamics of Underdamped Stochastic Systems, Phys. Rev. Lett. 125, 058103 (2020).

[109] D. B. Brückner, N. Arlt, A. Fink, P. Ronceray, J. O. Rädler, and C. P. Broedersz, Learning the Dynamics of Cell-Cell Interactions in Confined Cell Migration, Proc. Natl. Acad. Sci. U.S.A. 118, e2016602118 (2021).

[110] M. M. Norton, P. Grover, M. F. Hagan, and S. Fraden, Optimal Control of Active Nematics, Phys. Rev. Lett. 125, 178005 (2020).

[111] J. Colen, M. Han, R. Zhang, S. A. Redford, L. M. Lemma, L. Morgan, P. V. Ruijgrok, R. Adkins, Z. Bryant, Z. Dogic et al., Machine Learning Active-Nematic Hydrodynamics, Proc. Natl. Acad. Sci. U.S.A. 118, e2016708118 (2021).

[112] Z. Zhou, C. Joshi, R. Liu, M. M. Norton, L. Lemma, Z. Dogic, M. F. Hagan, S. Fraden, and P. Hong, Machine Learning Forecasting of Active Nematics, Soft Matter 17, 738 (2021). 
[113] H. Jeckel, E. Jelli, R. Hartmann, P. K. Singh, R. Mok, J. F. Totz, L. Vidakovic, B. Eckhardt, J. Dunkel, and K. Drescher, Learning the Space-Time Phase Diagram of Bacterial Swarm Expansion, Proc. Natl. Acad. Sci. U.S.A. 116, 1489 (2019).

[114] A. Borzou, A. E. Patteson, and J. M. Schwarz, A DataDriven Statistical Description for the Hydrodynamics of Active Matter, New J. Phys. 23, 103004 (2021).

[115] A. R. Dulaney and J.F. Brady, Machine Learning for Phase Behavior in Active Matter Systems, Soft Matter 17, 6808 (2021).

[116] W. F. Paxton, K. C. Kistler, C. C. Olmeda, A. Sen, S. K. S. Angelo, Y. Cao, T. E. Mallouk, P. E. Lammert, and V. H. Crespi, Catalytic Nanomotors: Autonomous Movement of Striped Nanorods, J. Am. Chem. Soc. 126, 13424 (2004).

[117] D. Needleman and Z. Dogic, Active Matter at the Interface between Materials Science and Cell Biology, Nat. Rev. Mater. 2, 17048 (2017).

[118] S. A. Mallory, C. Valeriani, and A. Cacciuto, An Active Approach to Colloidal Self-Assembly, Annu. Rev. Phys. Chem. 69, 59 (2018).

[119] M. Nguyen, Y. Qiu, and S. Vaikuntanathan, Organization and Self-Assembly away from Equilibrium: Toward Thermodynamic Design Principles, Annu. Rev. Condens. Matter Phys. 12, 273 (2021).

[120] R. Golestanian, T. B. Liverpool, and A. Ajdari, Propulsion of a Molecular Machine by Asymmetric Distribution of Reaction Products, Phys. Rev. Lett. 94, 220801 (2005).

[121] R. Soto and R. Golestanian, Self-Assembly of Catalytically Active Colloidal Molecules: Tailoring Activity through Surface Chemistry, Phys. Rev. Lett. 112, 068301 (2014).

[122] B. Nasouri and R. Golestanian, Exact Phoretic Interaction of Two Chemically Active Particles, Phys. Rev. Lett. 124, 168003 (2020).

[123] Z. You, A. Baskaran, and M. C. Marchetti, Nonreciprocity as a Generic Route to Traveling States, Proc. Natl. Acad. Sci. U.S.A. 117, 19767 (2020).

[124] S. Saha, J. Agudo-Canalejo, and R. Golestanian, Scalar Active Mixtures: The Nonreciprocal Cahn-Hilliard Model, Phys. Rev. X 10, 041009 (2020).

[125] S. Saha, S. Ramaswamy, and R. Golestanian, Pairing, Waltzing and Scattering of Chemotactic Active Colloids, New J. Phys. 21, 063006 (2019).

[126] R. Matas-Navarro, R. Golestanian, T. B. Liverpool, and S. M. Fielding, Hydrodynamic Suppression of Phase Separation in Active Suspensions, Phys. Rev. E 90, 032304 (2014).

[127] N. Gravish, G. Gold, A. Zangwill, M. A. D. Goodisman, and D. I. Goldman, Glass-like Dynamics in Confined and Congested Ant Traffic, Soft Matter 11, 6552 (2015).

[128] J. Aguilar, D. Monaenkova, V. Linevich, W. Savoie, B. Dutta, H.-S. Kuan, M. D. Betterton, M. A. D. Goodisman, and D. I. Goldman, Collective Clog Control: Optimizing Traffic Flow in Confined Biological and Robophysical Excavation, Science 361, 672 (2018).

[129] Y. Ozkan-Aydin and D. I. Goldman, Self-Reconfigurable Multilegged Robot Swarms Collectively Accomplish Challenging Terradynamic Tasks, Sci. Robot. 6, eabf1628 (2021).

[130] P. Pietzonka, É. Fodor, C. Lohrmann, M. E. Cates, and U. Seifert, Autonomous Engines Driven by Active Matter:
Energetics and Design Principles, Phys. Rev. X 9, 041032 (2019).

[131] É. Fodor, T. Nemoto, and S. Vaikuntanathan, Dissipation Controls Transport and Phase Transitions in Active Fluids: Mobility, Diffusion and Biased Ensembles, New J. Phys. 22, 013052 (2020).

[132] É. Fodor, R. L. Jack, and M. E. Cates, Irreversibility and Biased Ensembles in Active Matter: Insights from Stochastic Thermodynamics, arXiv:2104.06634.

[133] S. Saha, R. Golestanian, and S. Ramaswamy, Clusters, Asters, and Collective Oscillations in Chemotactic Colloids, Phys. Rev. E 89, 062316 (2014).

[134] A. Gelimson, K. Zhao, C. K. Lee, W. T. Kranz, G. C. L. Wong, and R. Golestanian, Multicellular Self-Organization of $P$. aeruginosa due to Interactions with Secreted Trails, Phys. Rev. Lett. 117, 178102 (2016).

[135] K. Husain and M. Rao, Emergent Structures in an Active Polar Fluid: Dynamics of Shape, Scattering, and Merger, Phys. Rev. Lett. 118, 078104 (2017).

[136] H. R. O. Sohn, C. D. Liu, and I. I. Smalyukh, Schools of Skyrmions with Electrically Tunable Elastic Interactions, Nat. Commun. 10, 1 (2019).

[137] C. Collinet and T. Lecuit, Programmed and Self-Organized Flow of Information during Morphogenesis, Nat. Rev. Mol. Cell Biol. 22, 245 (2021).

[138] P. Gross, K. V. Kumar, and S. W. Grill, How Active Mechanics and Regulatory Biochemistry Combine to Form Patterns in Development, Annu. Rev. Biophys. 46, 337 (2017).

[139] E. Frey and F. Brauns, Self-Organisation of Protein Patterns, arXiv:2012.01797.

[140] G. Frangipane, D. Dell'Arciprete, S. Petracchini, C. Maggi, F. Saglimbeni, S. Bianchi, G. Vizsnyiczai, M. L. Bernardini, and R. Di Leonardo, Dynamic Density Shaping of Photokinetic E. coli, eLife 7, e36608 (2018).

[141] J. Arlt, V. A. Martinez, A. Dawson, T. Pilizota, and W. C. K. Poon, Painting with Light-Powered Bacteria, Nat. Commun. 9, 768 (2018).

[142] M. C. Wigbers, T. H. Tan, F. Brauns, J. Liu, S. Z. Swartz, E. Frey, and N. Fakhri, A Hierarchy of Protein Patterns Robustly Decodes Cell Shape Information, Nat. Phys. 17, 578 (2021).

[143] U. Khadka, V. Holubec, H. Yang, and F. Cichos, Active Particles Bound by Information Flows, Nat. Commun. 9, 3864 (2018).

[144] D. Geiss, K. Kroy, and V. Holubec, Brownian Molecules Formed by Delayed Harmonic Interactions, New J. Phys. 21, 093014 (2019).

[145] F. A. Lavergne, H. Wendehenne, T. Bäuerle, and C. Bechinger, Group Formation and Cohesion of Active Particles with Visual Perception-Dependent Motility, Science 364, 70 (2019).

[146] D. Dasgupta, D. Pally, D. K. Saini, R. Bhat, and A. Ghosh, Nanomotors Sense Local Physicochemical Heterogeneities in Tumor Microenvironments, Angew. Chem., Int. Ed. Engl. 59, 23690 (2020).

[147] S. A. M. Loos and S. H. L. Klapp, Irreversibility, Heat and Information Flows Induced by Non-reciprocal Interactions, New J. Phys. 22, 123051 (2020). 
[148] G. Wang, T. V. Phan, S. Li, M. Wombacher, J. Qu, Y. Peng, G. Chen, D. I. Goldman, S. A. Levin, R. H. Austin, and L. Liu, Emergent Field-Driven Robot Swarm States, Phys. Rev. Lett. 126, 108002 (2021).

[149] E. Tang and R. Golestanian, Quantifying Configurational Information for a Stochastic Particle in a Flow-Field, New J. Phys. 22, 083060 (2020).

[150] P. Chugh and E. K. Paluch, The Actin Cortex at a Glance, J. Cell Sci. 131, jcs186254 (2018).

[151] T. H. Tan, M. Malik-Garbi, E. Abu-Shah, J. Li, A. Sharma, F. C. MacKintosh, K. Keren, C. F. Schmidt, and N. Fakhri, Self-Organized Stress Patterns Drive State Transitions in Actin Cortices, Sci. Adv. 4, eaar2847 (2018).

[152] K. E. Sawin, K. LeGuellec, M. Philippe, and T. J. Mitchison, Mitotic Spindle Organization by a Plus-End-Directed Microtubule Motor, Nature (London) 359, 540 (1992).

[153] J. Brugués and D. Needleman, Physical Basis of Spindle Self-Organization, Proc. Natl. Acad. Sci. U.S.A. 111, 18496 (2014).

[154] L. Tociu, É. Fodor, T. Nemoto, and S. Vaikuntanathan, How Dissipation Constrains Fluctuations in Nonequilibrium Liquids: Diffusion, Structure, and Biased Interactions, Phys. Rev. X 9, 041026 (2019).

[155] N. Roy, N. Leroux, A. K. Sood, and R. Ganapathy, Tuning the Performance of a Micrometer-Sized Stirling Engine through Reservoir Engineering, Nat. Commun. 12, 4927 (2021).

[156] S. Krishnamurthy, S. Ghosh, D. Chatterji, R. Ganapathy, and A. K. Sood, A Micrometre-Sized Heat Engine Operating between Bacterial Reservoirs, Nat. Phys. 12, 1134 (2016).

[157] T. Ekeh, M.E. Cates, and É. Fodor, Thermodynamic Cycles with Active Matter, Phys. Rev. E 102, 010101(R) (2020).

[158] G. Falasco, R. Rao, and M. Esposito, Information Thermodynamics of Turing Patterns, Phys. Rev. Lett. 121, 108301 (2018).

[159] U. Seifert, Stochastic Thermodynamics, Fluctuation Theorems and Molecular Machines, Rep. Prog. Phys. 75, 126001 (2012).

[160] H.-M. Chun, Q. Gao, and J. M. Horowitz, Nonequilibrium Green-Kubo Relations for Hydrodynamic Transport from an Equilibrium-like Fluctuation-Response Equality, arXiv:2103.09288.

[161] M. Han, M. Fruchart, C. Scheibner, S. Vaikuntanathan, W. Irvine, J. de Pablo, and V. Vitelli, Statistical Mechanics of a Chiral Active Fluid, arXiv:2002.07679.

[162] J. M. Horowitz and T. R. Gingrich, Thermodynamic Uncertainty Relations Constrain Non-equilibrium Fluctuations, Nat. Phys. 16, 15 (2020).

[163] J. Li, J. M. Horowitz, T. R. Gingrich, and N. Fakhri, Quantifying Dissipation Using Fluctuating Currents, Nat. Commun. 10, 1666 (2019).

[164] T. H. Tan, A. Mietke, H. Higinbotham, J. Li, Y. Chen, P. J. Foster, S. Gokhale, J. Dunkel, and N. Fakhri, Development Drives Dynamics of Living Chiral Crystals, arXiv: 2105.07507.

[165] D. Mizuno, C. Tardin, C. F. Schmidt, and F. C. MacKintosh, Nonequilibrium Mechanics of Active Cytoskeletal Networks, Science 315, 370 (2007).
[166] S.-W. Wang, K. Kawaguchi, S.-I. Sasa, and L.-H. Tang, Entropy Production of Nanosystems with Time Scale Separation, Phys. Rev. Lett. 117, 070601 (2016).

[167] L. P. Dadhichi, A. Maitra, and S. Ramaswamy, Origins and Diagnostics of the Nonequilibrium Character of Active Systems, J. Stat. Mech. (2018) 123201.

[168] A. J. Levine and F. C. MacKintosh, The Mechanics and Fluctuation Spectrum of Active Gels, J. Phys. Chem. B 113, 3820 (2009).

[169] J. Prost, J.-F. Joanny, and J. M. R. Parrondo, Generalized Fluctuation-Dissipation Theorem for Steady-State Systems, Phys. Rev. Lett. 103, 090601 (2009).

[170] N. Fakhri, A. D. Wessel, C. Willms, M. Pasquali, D. R. Klopfenstein, F. C. MacKintosh, and C. F. Schmidt, HighResolution Mapping of Intracellular Fluctuations Using Carbon Nanotubes, Science 344, 1031 (2014).

[171] L. Dinis, P. Martin, J. Barral, J. Prost, and J. F. Joanny, Fluctuation-Response Theorem for the Active Noisy Oscillator of the Hair-Cell Bundle, Phys. Rev. Lett. 109, 160602 (2012).

[172] J. Liphardt, S. Dumont, S. B. Smith, I. Tinoco, and C. Bustamante, Equilibrium Information from Nonequilibrium Measurements in an Experimental Test of Jarzynski's Equality, Science 296, 1832 (2002).

[173] R. Kawai, J. M. R. Parrondo, and C. Van den Broeck, Dissipation: The Phase-Space Perspective, Phys. Rev. Lett. 98, 080602 (2007).

[174] É. Roldán and J. M. R. Parrondo, Entropy Production and Kullback-Leibler Divergence between Stationary Trajectories of Discrete Systems, Phys. Rev. E 85, 031129 (2012).

[175] J. M. R. Parrondo, C. Van den Broeck, and R. Kawai, Entropy Production and the Arrow of Time, New J. Phys. 11, 073008 (2009).

[176] A. Gomez-Marin, J. M. R. Parrondo, and C. Van den Broeck, The Footprints of Irreversibility, Europhys. Lett. 82, 50002 (2008).

[177] J. M. Horowitz and C. Jarzynski, Illustrative Example of the Relationship between Dissipation and Relative Entropy, Phys. Rev. E 79, 021106 (2009).

[178] É. Roldán, J. Barral, P. Martin, J. M. R. Parrondo, and F. Jülicher, Arrow of Time in Active Fluctuations, arXiv: 1803.04743.

[179] I. A. Martínez, G. Bisker, J. M. Horowitz, and J. M. R. Parrondo, Inferring Broken Detailed Balance in the Absence of Observable Currents, Nat. Commun. 10, 3542 (2019).

[180] É. Fodor, C. Nardini, M. E. Cates, J. Tailleur, P. Visco, and F. van Wijland, How Far from Equilibrium Is Active Matter?, Phys. Rev. Lett. 117, 038103 (2016).

[181] T. H. Tan, G. A. Watson, Yu.-C. Chao, J. Li, T. R. Gingrich, J. M. Horowitz, and N. Fakhri, Scale-Dependent Irreversibility in Living Matter, arXiv:2107.05701.

[182] B. Guo, S. Ro, A. Shih, T. V. Phan, R. H. Austin, S. Martiniani, D. Levine, and P. M. Chaikin, Play. Pause. Rewind. Measuring Local Entropy Production and Extractable Work in Active Matter, arXiv:2105.12707.

[183] A. Seif, M. Hafezi, and C. Jarzynski, Machine Learning the Thermodynamic Arrow of Time, Nat. Phys. 17, 105 (2021). 
[184] C. Nardini, É. Fodor, E. Tjhung, F. van Wijland, J. Tailleur, and M. E. Cates, Entropy Production in Field Theories without Time-Reversal Symmetry: Quantifying the Nonequilibrium Character of Active Matter, Phys. Rev. X 7 , 021007 (2017).

[185] T. Frohoff-Hülsmann, J. Wrembel, and U. Thiele, Suppression of Coarsening and Emergence of Oscillatory Behavior in a Cahn-Hilliard Model with Nonvariational coupling, Phys. Rev. E 103, 042602 (2021).

[186] M. Fruchart, R. Hanai, P. B. Littlewood, and V. Vitelli, Non-reciprocal Phase Transitions, Nature (London) 592, 363 (2021).

[187] C. Scheibner, A. Souslov, D. Banerjee, P. Surowka, W. T. M. Irvine, and V. Vitelli, Odd Elasticity, Nat. Phys. 16, 475 (2020).

[188] A. V. Ivlev, J. Bartnick, M. Heinen, C.-R. Du, V. Nosenko, and H. Löwen, Statistical Mechanics where Newton's Third Law Is Broken, Phys. Rev. X 5, 011035 (2015).

[189] N. P. Kryuchkov, A. V. Ivlev, and S. O. Yurchenko, Dissipative Phase Transitions in Systems with Nonreciprocal Effective Interactions, Soft Matter 14, 9720 (2018).

[190] C. H. Meredith, P. G. Moerman, J. Groenewold, Yu.-J. Chiu, W. K. Kegel, A. van Blaaderen, and L. D. Zarzar, Predator-Prey Interactions between Droplets Driven by Non-reciprocal Oil Exchange, Nat. Chem. 12, 1136 (2020).

[191] M. Durve, A. Saha, and A. Sayeed, Active Particle Condensation by Non-reciprocal and Time-Delayed Interactions, Eur. Phys. J. E 41, 49 (2018).

[192] D. Helbing and P. Molnar, Social Force Model for Pedestrian Dynamics, Phys. Rev. E 51, 4282 (1995).

[193] H. Hong and S. H. Strogatz, Kuramoto Model of Coupled Oscillators with Positive and Negative Coupling Parameters: An Example of Conformist and Contrarian Oscillators, Phys. Rev. Lett. 106, 054102 (2011).

[194] E. Theveneau, B. Steventon, E. Scarpa, S. Garcia, X. Trepat, A. Streit, and R. Mayor, Chase-and-Run between Adjacent Cell Populations Promotes Directional Collective Migration, Nat. Cell Biol. 15, 763 (2013).

[195] L. Xiong, Y. Cao, R. Cooper, W.-J. Rappel, J. Hasty, and L. Tsimring, Flower-like Patterns in Multi-species Bacterial Colonies, eLife 9, e48885 (2020).

[196] M. A. Tsyganov, J. Brindley, A. V. Holden, and V. N. Biktashev, Quasisoliton Interaction of Pursuit-Evasion Waves in a Predator-Prey System, Phys. Rev. Lett. 91, 218102 (2003).

[197] L. Onsager, Reciprocal Relations in Irreversible Processes. I., Phys. Rev. 37, 405 (1931).

[198] D. Yanni, P. Márquez-Zacarías, P. J. Yunker, and W. C. Ratcliff, Drivers of Spatial Structure in Social Microbial Communities, Curr. Biol. 29, R545 (2019).

[199] A. I. Curatolo, N. Zhou, Y. Zhao, C. Liu, A. Daerr, J. Tailleur, and J. Huang, Engineering Cooperative Patterns in Multi-species Bacterial Colonies, bioRxiv:798827.

[200] C> M. Bender, Making Sense of Non-Hermitian Hamiltonians, Rep. Prog. Phys. 70, 947 (2007).

[201] V. Khemani, R. Moessner, and S. L. Sondhi, A Brief History of Time Crystals, arXiv:1910.10745.
[202] A. McDonald, R. Hanai, and A. A. Clerk, Non-equilibrium Stationary States of Quantum Non-Hermitian Lattice Models, arXiv:2103.01941.

[203] J. OByrne and J. Tailleur, Lamellar to Micellar Phases and beyond: When Tactic Active Systems Admit Free Energy Functionals, Phys. Rev. Lett. 125, 208003 (2020).

[204] R. K. Gupta, R. Kant, H. Soni, A. K. Sood, and S. Ramaswamy, Active Nonreciprocal Attraction between Motile Particles in an Elastic Medium, arXiv:2007.04860.

[205] K. Kruse, J.-F. Joanny, F. Jülicher, J. Prost, and K. Sekimoto, Generic Theory of Active Polar Gels: A Paradigm for Cytoskeletal Dynamics, Eur. Phys. J. E 16, 5 (2005).

[206] S. Ramaswamy, Active Matter, J. Stat. Mech. (2017) 054002.

[207] L. P. Dadhichi, J. Kethapelli, R. Chajwa, S. Ramaswamy, and A. Maitra, Nonmutual Torques and the Unimportance of Motility for Long-Range Order in Two-Dimensional Flocks, Phys. Rev. E 101, 052601 (2020).

[208] T. Markovich, É. Fodor, E. Tjhung, and M. E. Cates, Thermodynamics of Active Field Theories: Energetic Cost of Coupling to Reservoirs, Phys. Rev. X 11, 021057 (2021).

[209] B. Kelvin and W. Thomson, The Molecular Tactics of a Crystal (Clarendon, Oxford, 1894).

[210] L. Pasteur, Sur les Relations qui Peuvent Exister entre la Forme Crystalline, la Composition Chimique et le Sens de la Polarization Rotatoire, Ann. Chim. Phys. 24, 442 (1848).

[211] T. C. Lubensky, A. B. Harris, R. D. Kamien, and G. Yan, Chirality in Liquid Crystals: From Microscopic Origins to Macroscopic Structure, Ferroelectrics 212, 1 (1998).

[212] A. B. Harris, R. D. Kamien, and T. C. Lubensky, Molecular Chirality and Chiral Parameters, Rev. Mod. Phys. 71, 1745 (1999).

[213] E. Efrati and W. T. M. Irvine, Orientation-Dependent Handedness and Chiral Design, Phys. Rev. X 4, 011003 (2014).

[214] G. Dietler, R. Kusner, W. Kusner, E. Rawdon, and P. Szymczak, Chirality for Crooked Curves, arXiv: 2004.10338.

[215] T. C. Lubensky, Hydrodynamics of Cholesteric Liquid Crystals, Phys. Rev. A 6, 452 (1972).

[216] L. Radzihovsky and T. C. Lubensky, Nonlinear Smectic Elasticity of Helical State in Cholesteric Liquid Crystals and Helimagnets, Phys. Rev. E 83, 051701 (2011).

[217] A. Maitra and S. Ramaswamy, Oriented Active Solids, Phys. Rev. Lett. 123, 238001 (2019).

[218] T. C. Adhyapak, S. Ramaswamy, and J. Toner, Live Soap: Stability, Order, and Fluctuations in Apolar Active Smectics, Phys. Rev. Lett. 110, 118102 (2013).

[219] S. J. Kole, G. P. Alexander, S. Ramaswamy, and A. Maitra, Layered Chiral Active Matter: Beyond Odd Elasticity, Phys. Rev. Lett. 126, 248001 (2021).

[220] H. Löwen, Chirality in Microswimmer Motion: From Circle Swimmers to Active Turbulence, Eur. Phys. J. Special Topics 225, 2319 (2016).

[221] B. Liebchen and D. Levis, Collective Behavior of Chiral Active Matter: Pattern Formation and Enhanced Flocking, Phys. Rev. Lett. 119, 058002 (2017). 
[222] B. C. van Zuiden, J. Paulose, W. T. M. Irvine, D. Bartolo, and V. Vitelli, Spatiotemporal Order and Emergent Edge Currents in Active Spinner Materials, Proc. Natl. Acad. Sci. U.S.A. 113, 12919 (2016).

[223] J. L. A. Gomez, J. P. Steimel, and A. Alexander-Katz, Elasticity-Induced Force Reversal between Active Spinning Particles in Dense Passive Media, Nat. Commun. 7, 11325 (2016).

[224] J.-C. Tsai, F. Ye, J. Rodriguez, J. P. Gollub, and T. C. Lubensky, A Chiral Granular Gas, Phys. Rev. Lett. 94, 214301 (2005).

[225] S. Ahmad, S. B. Routh, V. Kamarthapu, J. Chalissery, S. Muthukumar, T. Hussain, S. P. Kruparani, M. V. Deshmukh, and R. Sankaranarayanan, Mechanism of Chiral Proofreading during Translation of the Genetic Code, eLife 2, e01519 (2013).

[226] P. Arora, A. K. Sood, and R. Ganapathy, Emergent Stereoselective Interactions and Self-Recognition in Polar Chiral Active Ellipsoids, Sci. Adv. 7, eabd0331 (2021).

[227] S. Fürthauer, M. Strempel, S. W. Grill, and F. Jülicher, Active Chiral Fluids, Eur. Phys. J. E 35, 89 (2012).

[228] S. Fürthauer, M. Strempel, S. W. Grill, and F. Jülicher, Active Chiral Processes in Thin Films, Phys. Rev. Lett. 110, 048103 (2013).

[229] T. Markovich, E. Tjhung, and M. E. Cates, Chiral Active Matter: Microscopic Torque Dipoles Have More than One Hydrodynamic Description, New J. Phys. 21, 112001 (2019).

[230] A. Maitra and M. Lenz, Spontaneous Rotation Can Stabilise Ordered Chiral Active Fluids, Nat. Commun. 10, 920 (2019).

[231] S. R. Naganathan, S. Fürthauer, M. Nishikawa, F. Jülicher, and S. W. Grill, Active Torque Generation by the Actomyosin Cell Cortex Drives Left-Right Symmetry Breaking, eLife 3, e04165 (2014).

[232] L. G. Pimpale, T. C. Middelkoop, A. Mietke, and S. W. Grill, Cell Lineage-Dependent Chiral Actomyosin Flows Drive Cellular Rearrangements in Early Caenorhabditis elegans Development, eLife 9, e54930 (2020).

[233] D. Banerjee, V. Vitelli, F. Jülicher, and P. Surówka, Active Viscoelasticity of Odd Materials, Phys. Rev. Lett. 126, 138001 (2021).

[234] W. Helfrich, Deformation of Cholesteric Liquid Crystals with Low Threshold Voltage, Appl. Phys. Lett. 17, 531 (1970).

[235] J. P. Hurault, Static Distortions of a Cholesteric Planar Structure Induced by Magnetic or ac Electric Fields, J. Chem. Phys. 59, 2068 (1973).

[236] C. A. Whitfield, T. C. Adhyapak, A. Tiribocchi, G. P. Alexander, D. Marenduzzo, and S. Ramaswamy, Hydrodynamic Instabilities in Active Cholesteric Liquid Crystals, Eur. Phys. J. E 40, 50 (2017).

[237] T. Gibaud, C. N. Kaplan, P. Sharma, M. J. Zakhary, A. Ward, R. Oldenbourg, R. B. Meyer, R. D. Kamien, T. R. Powers, and Z. Dogic, Achiral Symmetry Breaking and Positive Gaussian Modulus Lead to Scalloped Colloidal Membranes, Proc. Natl. Acad. Sci. U.S.A. 114, E3376 (2017).
[238] T. Markovich and T. C. Lubensky, Odd Viscosity in Active Matter: Microscopic Origin and 3D Effects, Phys. Rev. Lett. 127, 048001 (2021).

[239] K. W. Rogers and A. F. Schier, Morphogen Gradients: From Generation to Interpretation, Annu. Rev. Cell Dev. Biol. 27, 377 (2011).

[240] J. Prost, F. Jülicher, and J.-F. Joanny, Active Gel Physics, Nat. Phys. 11, 111 (2015).

[241] S. J. Streichan, M. F. Lefebvre, N. Noll, E. F. Wieschaus, and B. I. Shraiman, Global Morphogenetic Flow Is Accurately Predicted by the Spatial Distribution of Myosin Motors, eLife 7, e27454 (2018).

[242] R. G. Morris and M. Rao, Active Morphogenesis of Epithelial Monolayers, Phys. Rev. E 100, 022413 (2019).

[243] G. Struhl, J. Casal, and P. A. Lawrence, Dissecting the Molecular Bridges That Mediate the Function of Frizzled in Planar Cell Polarity, Development 139, 3665 (2012).

[244] L. Balasubramaniam, A. Doostmohammadi, T. B. Saw, G. H. N. S. Narayana, R. Mueller, T. Dang, M. Thomas, S. Gupta, S. Sonam, A. S. Yap et al., Investigating the Nature of Active Forces in Tissues Reveals How Contractile Cells Can Form Extensile Monolayers, Nat. Mater. 20, 1156 (2021).

[245] X. Serra-Picamal, V. Conte, R. Vincent, E. Anon, D. T. Tambe, E. Bazellieres, J. P. Butler, J. J. Fredberg, and X. Trepat, Mechanical Waves during Tissue Expansion, Nat. Phys. 8, 628 (2012).

[246] R. Alert and X. Trepat, Physical Models of Collective Cell Migration, Annu. Rev. Condens. Matter Phys. 11, 77 (2020).

[247] C. Duclut, J. Paijmans, M. M. Inamdar, C. D. Modes, and F. Jülicher, Nonlinear Rheology of Cellular Networks, arXiv:2103.16462.

[248] Z. Pujic and J. Malicki, in Seminars in Cell \& Developmental Biology (Elsevier, New York, 2004), Vol. 15, pp. 105-114.

[249] A.-K. Classen, K. I. Anderson, E. Marois, and S. Eaton, Hexagonal Packing of Drosophila Wing Epithelial Cells by the Planar Cell Polarity Pathway, Dev. Cell 9, 805 (2005).

[250] D. A. Sun and N. H. Patel, The Amphipod Crustacean Parhyale hawaiensis: An Emerging Comparative Model of Arthropod Development, Evolution, and Regeneration, Wiley Interdiscip. Rev.: Dev. Biol. 8, e355 (2019).

[251] G. Salbreux, L. K. Barthel, P. A. Raymond, and D. K. Lubensky, Coupling Mechanical Deformations and Planar Cell Polarity to Create Regular Patterns in the Zebrafish Retina, PLoS Comput. Biol. 8, e1002618 (2012).

[252] R. Etournay, M. Merkel, M. Popovi, H. Brandl, N. A. Dye, B. Aigouy, G. Salbreux, S. Eaton, and F. Jlicher, Tissueminer: A Multiscale Analysis Toolkit to Quantify How Cellular Processes Create Tissue Dynamics, eLife 5, e14334 (2016).

[253] V. Ajeti, A. P. Tabatabai, A. J. Fleszar, M. F. Staddon, D. S. Seara, C. Suarez, M. S. Yousafzai, D. Bi, D. R. Kovar, S. Banerjee et al., Wound Healing Coordinates Actin Architectures to Regulate Mechanical Work, Nat. Phys. 15, 696 (2019).

[254] S. Camalet, T. Duke, F. Jülicher, and J. Prost, Auditory Sensitivity Provided by Self-Tuned Critical Oscillations of Hair Cells, Proc. Natl. Acad. Sci. U.S.A. 97, 3183 (2000). 
[255] W. Bialek, A. Cavagna, I. Giardina, T. Mora, E. Silvestri, M. Viale, and A. M. Walczak, Statistical Mechanics for Natural Flocks of Birds, Proc. Natl. Acad. Sci. U.S.A. 109, 4786 (2012).

[256] D. Krotov, J. O. Dubuis, T. Gregor, and W. Bialek, Morphogenesis at Criticality, Proc. Natl. Acad. Sci. U.S.A. 111, 3683 (2014).

[257] A. R. Milewski, D. Ó. Maoiléidigh, J. D. Salvi, and A. J. Hudspeth, Homeostatic Enhancement of Sensory Transduction, Proc. Natl. Acad. Sci. U.S.A. 114, E6794 (2017).

[258] M. Paoluzzi, M. Leoni, and M. C. Marchetti, Information and Motility Exchange in Collectives of Active Particles, Soft Matter 16, 6317 (2020).

[259] A. Norambuena, F.J. Valencia, and F. Guzmán-Lastra, Understanding Contagion Dynamics through Microscopic
Processes in Active Brownian Particles, Sci. Rep. 10, 20845 (2020).

[260] Y.Zhao, C. Huepe, and P. Romanczuk, Contagion Dynamics in Self-Organized Systems of Self-Propelled Agents, arXiv: 2103.12618.

[261] N. Ganai, S. Sengupta, and G. I. Menon, Chromosome Positioning from Activity-Based Segregation, Nucl. Acids Res. 42, 4145 (2014).

[262] A. Y. Grosberg and J.-F. Joanny, Nonequilibrium Statistical Mechanics of Mixtures of Particles in Contact with Different Thermostats, Phys. Rev. E 92, 032118 (2015).

[263] S. Shankar, A. Souslov, M.J. Bowick, M. C. Marchetti, and V. Vitelli, Topological Active Matter, arXiv:2010 .00364 . 